\title{
Latent cause inference during extinction learning in trauma-exposed individuals with and without PTSD
}

Agnes Norbury ${ }^{1 *}$, Hannah Brinkman ${ }^{1}$, Mary Kowalchyk ${ }^{1}$, Elisa Monti ${ }^{1}$, Robert H Pietrzak ${ }^{2,3}$, Daniela Schiller ${ }^{1,4}$, Adriana Feder ${ }^{1}$

${ }^{1}$ Department of Psychiatry, Icahn School of Medicine at Mount Sinai, New York, NY, USA

${ }^{2}$ Department of Psychiatry, Yale University School of Medicine, New Haven, CT, USA

${ }^{3}$ Unite States Department of Veterans Affairs National Center for Posttraumatic Stress Disorder, Clinical Neurosciences Division, VA Connecticut Healthcare System, West Haven, CT, USA

${ }^{4}$ Department of Neuroscience and Friedman Brain Institute, Icahn School of Medicine at Mount

Sinai, New York, NY, USA

*Corresponding author: Agnes Norbury, One Gustave L. Levy Place, Department of Psychiatry, Box 1230, New York, NY 10029, USA. Email: agnes.norbury@mssm.edu 


\section{Abstract}

\section{Background}

Problems in learning that sights, sounds, or situations that were once associated with danger have become safe (extinction learning) may explain why some individuals suffer prolonged psychological distress following traumatic experiences. Although simple learning models have been unable to provide a convincing account of why this learning fails, it has recently been proposed that this may be explained by individual differences in beliefs about the causal structure of the environment.

\section{Methods}

Here, we tested two competing hypotheses as to how differences in causal inference might be related to trauma-related psychopathology, using extinction learning data collected from clinically well-characterized individuals with varying degrees of post-traumatic stress $(N=56)$. Model parameters describing individual differences in causal inference were related to multiple PTSD and depression symptom dimensions via network analysis.

\section{Results}

Individuals with more severe PTSD were more likely to assign observations from conditioning and extinction stages to a single underlying cause. Specifically, network analysis revealed a negative relationship between tendency to infer multiple causes were active in the environment and reexperiencing symptom severity.

\section{Conclusions}

We interpret these results as providing evidence of a primary deficit in discriminative learning in participants with more severe PTSD symptoms. Specifically, a greater tendency to attribute all stimulus configurations to the same underlying cause resulted in greater uncertainty about stimulusoutcome associations, impeding learning both that certain stimuli were safe, and that certain stimuli were no longer dangerous. Better understanding of the role of causal inference in trauma-related psychopathology may have relevance for the refinement of cognitive therapies for these disorders. 


\section{Introduction}

Post-traumatic stress disorder (PTSD) can be thought of a disorder of inappropriate fear, driven by a failure to update expectations when objects or contexts that were once associated with danger become safe (Lissek \& van Meurs, 2015). However, simple associative accounts of learning are unable to convincingly account for why such fear persists - particularly in the face of prolonged exposure (extinction) training, or when considering relapse (spontaneous return of fear) (Dunsmoor et al., 2015; Levy \& Schiller, 2021). Recently, a novel computational account of extinction learning latent cause modelling - has been proposed by Gershman, Niv, and colleagues (Gershman et al., 2010; Gershman \& Niv, 2010, 2012). This account posits that during learning, individuals do not simply learn to associate different stimuli or contexts with outcomes, but rather that they attempt to draw inferences about the underlying environmental causes that are responsible for groups of observations (i.e., stimuli, context, and outcomes together). For example, an experimental animal may learn to infer that on different days, or when a different experimenter is present, painful stimuli are unlikely to be presented - rather than having to gradually update their stimulus-outcome associations during every new conditioning or extinction learning session. Individual differences in this inference process regulate whether an individual decides that the same cause is responsible for their current observations (and therefore that the original fear memory should be updated), or whether a new underlying cause is responsible (and therefore the original memory is left intact) (Gershman et al., 2017). According to this account, the inappropriate fear responses observed in post-traumatic stress syndromes could result from two different underlying mechanisms: 1) failure to retrieve a successfully formed extinction memory, as a result of inferring that a different cause is operating in the environment, or 2) failure to successfully form an extinction memory in the first place.

Computationally, the first case can be formalized as heightened tendency to segment ongoing experience into different causal clusters during extinction learning. Simulation evidence suggests that this would be reflected in faster learning during initial extinction training (due to lower conflict between conditioning and extinction trials), but greater vulnerability to relapse or spontaneous return of fear (e.g., if contextual cue changes mean that the old fear memory is retrieved, rather than the new extinction memory) (Gershman et al., 2010; Gershman \& Niv, 2012; Gershman et al., 2015). Indeed, tendency to infer more causes are active across conditioning and extinction episodes has been previously been shown to predict stronger return of physiological fear responses during next-day recall testing in healthy humans (Gershman \& Hartley, 2015).

However, a body of evidence also suggests that individuals with PTSD and other anxiety disorders show deficits in aversive processing that may be pre-requisites for successful extinction learning: in particular in the ability to discriminate between safe and danger-associated stimuli, in the context of potential aversive outcomes (pain or monetary loss). For example, both higher arousal to non pain/loss-associated stimuli during initial learning and greater physiological and self-reported aversion responses to all stimuli during extinction training are reliably observed in groups of individuals with anxiety disorders, compared to healthy controls (Duits et al., 2015; Marin et al., 2020). Further, heightened transfer of negative expectations to stimuli that are perceptually similar to fear-associated shapes or sounds has been observed in individuals with post-traumatic stress and 
anxiety (Lissek \& van Meurs, 2015; Kaczkurkin et al., 2016; Norbury et al., 2018). Intuitively, reduced ability to distinguish between (or poorer internal representation of) which stimuli were associated with which outcomes might result in a tendency to assign all observations to a single underlying cause. Importantly, a single underlying cause with poor distinction between different sets of observations could be reflected in both negative expectations for all stimuli (even those never associated with danger), and impeded extinction learning (due to greater uncertainty about stimulusoutcome configurations associated with that cause) (Gershman \& Niv, 2012). Therefore, it is possible that the inappropriate negative expectations associated with PTSD are the result of either heightened or reduced tendency to believe that different causes are responsible for observations during exposure to extinction.

Here, we sought to test these competing hypotheses by investigating latent cause inference during extinction learning in a group of clinically well-characterized trauma-exposed individuals with a range of experience of post-traumatic stress symptoms $(N=56)$. Specifically, we investigated whether trauma-exposed individuals with more severe PTSD symptoms would show a pattern of behaviour best explained by a greater or lower tendency to infer novel environmental causes, when compared to trauma-exposed individuals with less severe or no post-traumatic stress. We were particularly interested in whether differences in inference across aversive conditioning and extinction learning were related to individual difference in avoidance symptoms, as inappropriate avoidance behaviour is thought to be a core mechanism maintaining resistance to extinction in anxiety disorders (Arnaudova et al., 2017; Pittig et al., 2020), and there is some evidence that avoidance-related traits predict poorer response to cognitive therapy for PTSD (Badour et al., 2012; Békés et al., 2019). Following recent theoretical developments that favour modelling psychological disorders including post-traumatic stress as consisting of complex associations of interacting symptoms and other psychosocial factors (Borsboom, 2017), individual differences in latent cause inference were also related to multiple PTSD and depression symptom dimensions concurrently in an exploratory network analysis (see Greene et al., 2018; Armour et al., 2017; Fritz et al., 2018; de Haan et al., 2020).

The findings presented here represent the first evidence that individual differences in latent cause inference detected using a simple remotely-administered extinction learning paradigm are related to current psychological symptom severity. Ultimately, better understanding of how individual differences in causal inference contribute to maladaptive learning in anxiety and post-traumatic stress may have relevance for the refinement of cognitive and learning-based therapies for these disorders (Moutoussis et al., 2017). 


\section{Methods}

\section{Participants}

Participants were World Trade Center (WTC) disaster survivors and rescue/recovery workers, recruited from two ongoing studies at the Trauma and Resilience Program at the Icahn School of Medicine at Mount Sinai. All participants had DSM-5 Category A trauma exposure (defined as "actual or threatened death or serious injury", American Psychiatric Association, 2013) during the WTC disaster, as determined by clinical interview. Participants from both studies included individuals who currently met diagnostic criteria for full or subthreshold PTSD (for full inclusion/exclusion criteria see Supplementary Material), with one study also including traumaexposed individuals who were assessed as never having met criteria for PTSD. Both studies received ethical approval from the Institutional Review Board at the Icahn School of Medicine at Mount Sinai and all participants provided informed, written consent.

\section{Clinical and sociodemographic measures}

All participants completed an in-depth clinical interview with an experienced Trauma and Resilience Program team member. For $N=25$ participants this consisted of the Structure Clinical Interview for DSM-5 (SCID-5) and Clinician-Administered PTSD Scale for DSM-5 (CAPS-5) (Williams et al., 2015; Weathers, Blake, et al., 2013), and for $N=31$ participants this was the Mini-International Neuropsychiatric Interview for DSM-5 (MINI-5) (Sheehan et al., 1998). Additionally, participants completed the PTSD Checklist for DSM-5 (PCL-5) and The Beck Depression Inventory version II (BDI-II) self-report measures of PTSD and depression symptoms (Weathers, Litz, et al., 2013; Beck et al., 1996). PTSD symptoms were parsed into seven dimensions (re-experiencing, avoidance, negative affect, externalizing behaviour, anxious arousal, and dysphoric arousal symptoms clusters) which have been previously been demonstrated to provide the best account of symptoms data across multiple samples of trauma-exposed individuals (Armour et al., 2015, 2016). Depression symptoms as measured on the BDI-II were divided into 'cognitive' and 'physical/affective' subdimensions on the basis of results of a previous longitudinal analysis of diverse samples of depressed individuals (Bringmann et al., 2015). Information about lifetime trauma history and perceived levels of social support was also available (see Supplementary Material). A subset of individuals $(N=24)$ completed the Cogstate battery, a set of computerised tests probing general executive function that have been shown to be sensitive to mild cognitive impairment (Maruff et al., 2009).

\section{Extinction learning task}

Participants completed an extinction learning task, analogous in structure to that employed in a previous analysis of latent cause inference during extinction in healthy individuals (Gershman \& Hartley, 2015). This task consisted of three phases: an initial aversive conditioning phase (in context A), extinction learning phase (in context A), and further extinction learning (in novel context B) (Figure 1a). Importantly, the conditioned stimulus (CS) associated with the aversive loss outcome (US) - the CS + - was only partially reinforced $(P(\mathrm{US} \mid \mathrm{CS}+)=1 / 3)$, and the transition to extinction $(P(\mathrm{US} \mid \mathrm{CS}+)=0)$ was unsignalled. This design maximises uncertainty about whether $\mathrm{CS}+$ trials during extinction should be grouped with unreinforced conditioning phase CS + trials, implying a 
common cause is responsible for both kinds of observations, or instead that the change in contingencies indicates it is likely that a new cause is active in the environment. In order to test feasibility for future remote work, the extinction learning task was administered online (see Supplementary Material).

\section{Analysis}

Statistical analyses were carried out in R version 3.6.1 (R Core Team, 2019) and MATLAB R2019a (MathWorks Inc., 2019). Analysis code and version information for $\mathrm{R}$ packages is available at https://github.com/agnesnorbury/latent-cause-PTSD.

Extinction task data. Effects of within-task manipulations (effects of conditioned stimulus [CS] type and task stage) on loss expectancy ratings and response input times were explored using repeated-measures ANOVA (see Supplementary Material). Extinction resistance was defined as mean loss expectancy rating for the aversively conditioned CS (CS+) under extinction, measured at the end of both the initial extinction [context A] and further extinction learning [context B] task stages). Absolute values were used for CS + ratings (as opposed to difference in values between CS+ and non aversively-conditioned [CS-] stimuli), as - in contrast to other types of data such as GSR or BOLD signals - expectancy ratings have an absolute meaning. Further, experimental evidence suggests that individuals with PTSD may over-generalize negative information from conditioned to unconditioned stimuli (see Introduction) - which might result in inappropriately low differencebased values for these quantities (e.g., in the case where loss expectancy ratings are high for both CS+ and CS- stimuli). Since, across the group as a whole, 1) we observed maintained differential responding to CS+ and CS- stimuli at the end of initial extinction training (indicating incomplete learning), and 2) there were no obvious effects of the change-in-context manipulation on learning traces (Figure 1b), we did not investigate potential 'recall' effects (such as spontaneous recovery) between extinction trials at the end of context A and start of context B. Such an analysis may also be of limited validity here, as, in contrast to previous investigations (e.g., Gershman \& Hartley, 2015), there was no significant temporal delay between the two extinction training phases.

Latent cause modelling of extinction task data. Latent cause modelling of loss expectancy ratings data was carried out using code associated with Gershman \& Niv (2012) (https://github.com/sigershm/LCM). Following Gershman and Hartley (2015), latent cause modelling was applied to conditioning and initial extinction training data only. The last block of initial extinction learning trials was held out, so that model output would be unbiased by trials used to calculate extinction resistance both at the end of this stage [context A], and following further extinction learning [context B].

Briefly, the model assumes that the participant learns to associate groups of stimuli they observe, with different underlying states or causes. On each trial, participants compute the posterior probability that a given cause $c$ generated the observed configuration of stimuli (here, a 3D binary vector representing presence/absence of the CS+, CS-, and US), using Bayes' rule:

$$
P(\text { cause }=c \mid \text { stimuli }) \propto P(\text { stimuli } \mid \text { cause }=c) * P(\text { cause }=c)
$$

I.e., the inferred probability of an existing cause $c$ being active on a given trial, given observation of the trial stimuli, is proportional to the likelihood of that cause (consistency between current stimuli 
and prototypical stimulus configuration associated with cause $c$ ), multiplied by a prior term that indexes an individual's preference for simpler or more complex causal structures (Figure 2a). This prior biases the model to assign trials to a given cause in proportion to the number of trials previously assigned to that cause, and to a new cause with a probability proportional to the value of the free parameter alpha (i.e., the distribution over states is modelled using a Chinese Restaurant Process with concentration parameter $\alpha$ - see Gershman \& Niv, 2012; Gershman et al., 2015). Smaller values of $\alpha$ bias individuals towards simpler clusterings, where observations tend to be assigned to the same cause, and larger values towards more complex clusterings, where observations are assigned to different causes. As the learner has some uncertainty about the stimulus configuration associated with each cause, the output on each trial is a posterior probability distribution across potential underlying causes (each learner starts with an internal representation consisting of a single cause, and more causes are added as required by the model, up to a maximum limit). The model was fit to task data under a generative framework, by comparing how well models with a range of different $\alpha$ values could account for participants' task performance (see Supplementary Material).

The key output submitted to further analysis was the likelihood (for each participant) of a model where $\alpha$ was allowed to be $>0$ (i.e., with multiple inferred causes), compared to a model where $\alpha=0$ (single underlying cause), computed as a $\log$ Bayes Factor $(\log \mathrm{BF}) . \mathrm{A} \log \mathrm{BF} \geq 1$ is generally interpreted as representing strong evidence in favour of the comparator hypothesis (here, in favour of a multi-cause model) (Kass \& Raftery, 1995).

In order to assess goodness of fit, actual $v$ s predicted ratings generated by the model on each trial were compared for each participant using Pearson correlations. Permutation difference testing with 10,000 random assignments was used to compare goodness of fit $(r)$ values derived from the actual data to $N=1000$ randomly shuffled dummy datasets. Ability of the model to reliably recover $\alpha$ and $\operatorname{logBF}$ estimates from task data was assessed using simulation and recovery analysis (see Supplementary Material). Trial-by-trial associations between response times and internal model quantities were examined using linear mixed models, using the R package lmer'Test.

Bivariate relationships between latent cause inference and behavioural/clinical data. In order to account for individual differences in uncertainty about posterior estimates of the key internal model parameter $(\alpha), \log B F$ estimates were bivariately related to behavioural and clinical measures using weighted least squares regression, with regression weights set equal to peak of the posterior probability distribution over alpha values. As probability distributions sum to 1 , this peak value is inversely proportional to the width of spread of the probability density, which represents uncertainty about the posterior estimate (or how informative task data were in updating the uniform density prior). This ensures that greater weight in the analysis is allocated to estimates from participants for whom this quantity was more confidently derived (Figure S1b).

Network analysis of latent cause inference, clinical, and sociodemographic data. Network analysis was used to explore relationships between individual differences in latent cause inference and severity of multiple PTSD and depression symptom dimensions, whilst taking into account other relevant clinical and demographic factors. Specifically, networks were constructed using regularized Gaussian graphical estimation implemented in the R package qgraph, version 1.6.5 
(Epskamp et al., 2012). Under this approach, nodes represent observed variables, and connections between nodes (edges) represent unique pairwise associations (partial correlation coefficients) after conditioning on all other variables in the dataset. The application of regularization during estimation is intended to remove spurious connections from the network, such that any retained edges can be thought of as contributing meaningfully to the overall variance (according to simulation studies, at low ratios of number of observations to number of potential connections, edges discovered by this method are likely to represent edges in the true network, but some true edges may be missing; (Epskamp, 2018; Epskamp \& Fried, 2018; Williams et al., 2019). Stability of parameter estimation and power-related properties of the analysis were assessed via nonparametric bootstrap and simulation analyses, using functions from bootnet, version 1.4.3 (Epskamp et al., 2018). For full methodology (per Burger et al., 2020), see Supplementary Material. Extinction resistance (mean CS+ loss expectancy rating at the task) and safety learning failure (mean loss expectancy for the CS- at the end of the task), were included in the networks as well as $\log B F$ in order to ascertain if the latent cause model parameter was more closely related to symptoms than these simple behavioural performance indices (i.e., had greater explanatory power than behavioural differences alone). As per Armour et al. (2017), two nested networks were estimated: one consisting of PTSD/depression symptoms scores and behavioural task variables alone, and one with additional clinically-relevant covariates (age, education level, perceived level of social support, and additional lifetime trauma history).

\section{Results}

\section{Participants}

Demographic and clinical data for study participants are summarized in Table $1 . N=42(75 \%)$ participants currently met DSM-5 criteria for full or subthreshold WTC-related PTSD (mean PCL-5 total score 40.4 \pm 11.0$)$, and $N=14$ (25\%) participants were resilient to WTC trauma (no current or lifetime diagnosis of PTSD or other DSM-5 Axis-1 disorder; mean PCL-5 total score 1.6 \pm 1.7 ). Participants with full or subthreshold PTSD also reported moderate levels of depression symptoms

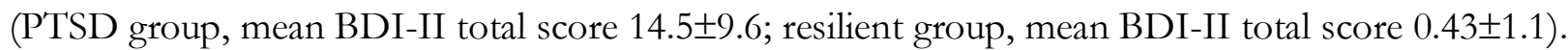
However, PTSD is known to be highly heterogenous (Armour et al., 2015; Contractor et al., 2017), and previous analyses in WTC responders and other populations have revealed reliable differences in patterns of covariance across symptom clusters (Pietrzak et al., 2014; Horn et al., 2016). In our sample, PTSD subscores exhibited continuous variation across participants (Table S1), with only moderate correlations observed between subscores (mean $r=0.67 \pm 0.16$; Figure S1) - justifying the use of multidimensional approach to PTSD symptomatology in our analysis.

\section{Extinction learning task}

Manipulation check. In order to check if participants performed as expected on the task, loss expectancy ratings and response times were analysed by repeated-measures ANOVA. Overall, participants entered greater loss expectancy ratings for the aversively conditioned (CS+) compared to non aversively-conditioned (CS-) stimuli, and decreased their ratings of CS+, but not CS-, stimuli 
over the course of the task (i.e., when these stimuli began to be presented in extinction) (Supplementary Results; Figure 1b). Participants responded more quickly at later stages in the task, but median response times remained $>3000 \mathrm{~ms}$, indicating preservation of relatively considered responding (Figure 1c).

\section{Latent cause modelling of extinction task data}

Model fit and validation. In order to assess how well the model accounted for our data, observed loss expectancy ratings on each trial were plotted against model-predicted output for each participant (Figure S2). Across participants, the mean correlation between actual and predicted loss expectancy ratings was 0.459 (SD 0.25). Permutation difference testing revealed that the mean $r$ value for actual $v$ s predicted loss ratings in our sample was significantly greater than that generated by fitting the same model to randomly shuffled data (mean $r$ for shuffled data $=0.118$; difference $=0.312, p<0.001$; Figure S3). Goodness-of-fit $(r)$ values did not differ between PTSD and resilient individuals (PTSD group, mean $r=0.477$; resilient group, mean $r=0.405$; $p>0.4$, Welch's twosample $t$ test), and were not related to $\operatorname{logBF}$ values ( $p>0.8$, Spearman's rank correlation test).

Simulation and recovery analysis revealed good parameter estimate stability and identifiability for task data (correlation between simulated and recovered alpha values $=0.838, p<0.001$; correlation with recovered beta values $=0.053, p>0.4$ ) (Figure S4a). Comparison of recovered logBF estimates for datasets simulated with $\alpha=0 v s \alpha>0$ revealed significantly different likelihood estimates $\left(t_{273}=-111\right.$, 95\%CI -3.70 - -3.84, $p<0.001$; Welch's two-sample $t$ test); with datasets simulated with $\alpha=0$ favouring a single cause model (mean $\log B F=-1.03 \pm 0.01$ ), and datasets simulated with $\alpha>0$ favouring a multi-cause model (mean $\log \mathrm{BF}=2.74 \pm 0.17$ ) (Figure $\mathrm{S} 4 \mathrm{~b})$.

Looking inside the model. Inspection of the posterior distribution over latent causes for all subjects indicated that participants mainly assigned observations to one or two latent causes (Figure 2c; across participants, marginal probability of a third cause was 0.117$)$. In order to visualise differences in behaviour associated with model output, participants were divided into two groups, defined according whether their behaviour provided strong evidence in favour of a multi- (vs single) cause model $(\log B F \geq 1$ vs $\log B F<1)$. Similar to Gershman and Hartley (2015), individuals whose responses provided no strong evidence in support of a multi-cause account appeared to learn more slowly across both conditioning and extinction stages (shallower curves for participants with $\log \mathrm{BF}<1$, Figure $2 \mathrm{~d}$ ). Formal comparison by fitting a simple linear slope to CS+ loss expectancy ratings over the course of the modelled period revealed significantly shallower gradients in the lower $\log \mathrm{BF}$ group $(\log \mathrm{BF}<1$, mean gradient $=-2.67 \pm 8.5 ; \log \mathrm{BF} \geq 1$ mean gradient $=-7.21 \pm 7.1 ; p=0.034$, Wilcoxon signed-rank test).

Notably, the lower logBF group also appeared to discriminate less between conditioned (lossassociated) and unconditioned (non loss-associated) stimuli. Over the course of the modelled period, individuals with lower $\log \mathrm{BF}$ values distinguished less between CS+ and CS- stimuli in terms of their loss expectancy ratings $(\log B F<1$, mean difference in rating $=18.2 \pm 24.7 ; \log B F \geq 1$ mean difference in rating $=43.4 \pm 27.0 ; p<0.001$, Wilcoxon signed-rank test). This difference was driven by both lower loss expectancy ratings for loss-conditioned (CS+) stimuli, and higher expectancy ratings for non loss-associated (CS-) stimuli, in the lower $\log B F$ group $(\operatorname{logBF}<1$ : mean $C S+$ rating $=46.2 \pm 14.3$, 
mean CS - rating $=28.0 \pm 23.6 ; \log B F \geq 1$ : mean $C S+$ rating $=58.3 \pm 13.0$, mean $C S$ - rating $=15.0 \pm 21.5$; $p=0.002, p=0.035$, respectively; Wilcoxon signed-rank tests). This suggests that the slower extinction learning in individuals with low evidence of a multi-cause model might be a result of more similar observation representations across different trial types (CS+ [reinforced], CS+ [unreinforced], and CS- trials) in these individuals (Figure 2b).

Importantly, simulated datasets where $\alpha$ was constrained to be close to or $>0$ were able to replicate this behavioural pattern: with the $\alpha \sim 0$ group (favouring a single latent cause model) showing both shallower gradients in CS + loss expectancy ratings and lower difference in ratings between CS + and CS- stimuli than the $\alpha>0$ group (favouring a multi-cause model), over the same task period (both $p<0.001$, Wilcoxon signed-rank tests) (Figure S5). This pattern was also robust to choice of $\operatorname{logBF}$ threshold used to define groups (Supplementary Material; Figure S6).

Relationship to extinction resistance and safety learning. $\log B F$ values were not related to extinction resistance (mean residual CS + loss expectancy rating) at the end of the modelled period, or end of the initial extinction learning stage (block $5, \beta=-4.2, p=0.267$; block $6, \beta=-6.7, p=0.132$; linear regressions weighted by certainty in posterior $\alpha$ estimate), but were significantly related to extinction resistance at the end of the task (block $9, \beta=-11.9, p=0.017$; Figure 2e, Figure S7a). This suggests that latent cause inference during initial learning might predict future resistance to extinction training - with higher likelihood of inferring a single cause during initial learning associated with persistence of loss expectancy for CS + stimuli many trials into extinction. Interestingly, $\log \mathrm{BF}$ values were also significantly negatively associated with CS- ratings at these three task stages $(\beta=-11.5,-9.9,-9.2$ for block 5, block 6 , block 9 , respectively; all $p<0.020)$, although these associations appear substantially non-linear (Figure S7b). This suggests that latent cause inference may also relate to either heightened generalization of aversive consequences from CS + to CS- stimuli, or failure of discriminative safety learning for CS-stimuli, over the course of the task.

An alternative explanation is that these relationships are due to a common non-specific effect, such as poorer working memory function, lower attentional performance, or more perseverative response style in individuals with lower $\log \mathrm{BF}$ estimates. In order to test this hypothesis, we used data from the Cogstate neurocognitive test battery that were available for a subset of participants. Although this test is likely underpowered $(N=24)$, we found no evidence of a relationship between Cogstate composite scores and $\log B F$ estimates $(\beta=0.010, p=0.338)$. Values of the scaling parameter $\beta$, which may reflect individual differences in use of the response scale, were unrelated to extinction resistance or failure of safety learning at the end of the modelled period, end of the initial extinction learning stage, or end of the task ( $p>0.11$, Spearman's rank correlation tests).

Relationship to response times. In order to further test whether lower logBF scores might reflect tendency towards a stimulus-independent or inattentive response style, we also examined whether median response times during each stage of the task were related to $\log B F$ estimates. Interestingly, there was marginal evidence of a negative relationship between $\log \mathrm{BF}$ and median response times during conditioning and initial extinction learning stages $(\beta=-552, p=0.054 ; \beta=-469, p=0.055)$ - with longer median response times associated with lower logBF scores (during further extinction in novel context B: $\beta=-341, p=0.214$; Figure S7c). This may indicate greater uncertainty about predicted values in lower $\log \mathrm{BF}$ individuals during initial learning and extinction training (Hyman, 1953). 
To examine this relationship more precisely, we analysed trial-by-trial variance in RT as a function of uncertainty over underlying latent causes and $\log B F$ estimates, in linear mixed models controlling for time-on-task (trial number) and expected value (predicted probability of loss) (cf Brown et al., 2018). Uncertainty about active causes was approximated by taking the maximum over the posterior probability distribution across causes on each trial, which is directly proportional to certainty that the most likely cause was responsible for trial observations. We found that response times were slower for individuals with lower logBF estimates ( $\beta=-1395[\mathrm{SE} 370] \mathrm{ms}, p<0.001)$, and on trials with greater likelihood of a single particular cause $(\beta=9457[\mathrm{SE} 456] \mathrm{ms}, p<0.001)$. Results were unchanged if the time-on-task effect was modelled as an exponential decay function, as suggested by Figure 1c (see Supplementary Material). This finding is consistent with slower RTs reflecting greater uncertainty about the configuration of observations associated with a particular cause (greater variance in prototypical stimulus and outcome vectors associated with that cause), rather than greater uncertainty about which cause was active on a given trial - in particular for individuals with greater tendency to group all observations as being the results of a single cause.

\section{Relationship between latent cause inference and PTSD symptoms}

Relationship with avoidance symptoms. In individual linear regression models weighted by posterior certainty in alpha parameter estimates, $\log B F$ values were significantly negatively related to PCL-5 avoidance symptoms ( $\beta=-0.89, p=0.045)$, and non-significantly related to PCL-5 total symptom severity score $(\beta=-5.6, p=0.069)$, Figure 3 a. Specifically, individuals with lower $\log B F$ values, indicating greater likelihood of a single cause model across conditioning and extinction learning, reported greater levels of avoidance symptoms. In order to test for evidence of a nonspecific relationship between psychological symptom levels and parameter estimates, logBF values were also compared to BDI-II total depression symptom scores $(\beta=-1.9, p=0.234)$. Beta values were not related to PCL-5 avoidance symptoms, PCL-5 total score, or BDI-II total score $(p>0.3$, Spearman's rank correlation tests).

Network analysis of extinction task parameters, PTSD, and depression symptoms. Whilst accounting for individual differences in extinction resistance and safety learning failure at the end of the task, greater severity of PTSD re-experiencing symptoms was associated with lower logBF values (regularized edge weight -0.089 , bootstrapped 95\% CI for edge value $=-0.224-0$, Figure $3 \mathrm{~b}$ ). As reexperiencing symptoms were positively connected to avoidance symptoms (regularized edge weight 0.262 , bootstrapped $95 \% \mathrm{CI}=0.053-0.414$ ), this suggests that the relationship between $\operatorname{logBF}$ and avoidance behaviour may be mediated by more intense re-experiencing symptoms (intrusive thoughts, nightmares, flashbacks, and emotional and physiological reactivity to trauma-related cues). There was also a negative connection between $\log \mathrm{BF}$ and dysphoric arousal PTSD symptoms (difficulty concentrating and sleep disturbance; regularized edge weight -0.026, bootstrapped 95\%CI $=-0.153-0$; for sample weights and bootstrapped 95\%CIs for all network edges see Figure S8). Simulation-based power analysis revealed acceptable network recovery properties at $N=56$. Across 1000 simulations, the median correlation between true and recovered networks at this sample size was 0.765 (IQR 0.13). Median sensitivity (accurate discovery of present edges) was 0.694 (IQR 0.14), and specificity (accurate discovery of absent edges) was 0.767 (IQR 0.17). 
Network analysis incorporating other clinical and demographic covariates. When additional covariates (age, education level, cumulative trauma history, and perceived level of social support) were added to the network, the negative connection between $\log \mathrm{BF}$ values and re-experiencing symptoms was retained (regularized edge weight - 0.041 , bootstrapped 95\%CI for edge value - $0.175-$ 0 , Figure $3 \mathrm{c}$ ). There were also negative connections between age and lifetime trauma history and $\operatorname{logBF}$ scores. Specifically, individuals who were older, and participants who reported greater cumulative lifetime trauma tended to have lower logBF estimates (regularized edge weights -0.029, 0.020; bootstrapped 95\%CIs $-0.272-0,-0.234-0$; respectively; for sample weights and bootstrapped 95\%CIs for all edges see Figure S9). The overall structure of the network between PTSD/depression symptoms and extinction task variables was robust to inclusion of the additional covariates, as correlation between the edge weights derived from covariate controlled and noncovariate controlled networks was high (Spearman's $Q=0.955)$. However, simulation-based power analysis revealed that the structure of the full covariate-controlled network reported here should be interpreted with caution, as it is likely underpowered: with an $N$ of 150 or more required for satisfactory sensitivity and specificity in true network recovery.

\section{Discussion}

Here, we provide preliminary evidence that individual differences in latent cause inference, as measured during a simple behavioural extinction learning paradigm, may be related to experience of psychological symptoms following trauma. Specifically, we found that trauma-exposed individuals whose patterns of behavioural responses were associated with greater likelihood of a generative model with a single underlying cause exhibited greater resistance to extinction training in future trials, poorer safety learning, and higher levels of avoidance symptoms. In line with previous observations that, during the same measurement occasion, within-subjects deviations in internal avoidance symptoms are significantly associated with within-subjects deviations in occurrence of flashbacks (Greene et al., 2018; Hoffart et al., 2019), our exploratory cross-sectional network analysis incorporating multiple PTSD and depression symptom clusters indicated that the bivariate association between latent cause inference and avoidance may be mediated via greater severity of PTSD re-experiencing symptoms (intrusive thoughts, nightmares, flashbacks, and emotional/physiological reactivity to reminders). Importantly, this multivariate analysis also controlled for individual differences in task performance (extinction resistance and safety learning failure), indicating that the model-based index had additional explanatory power over raw behavioural scores with respect to prototypical post-traumatic symptoms.

Strengths of the data presented here include a clinically well-characterised sample: all participants completed an in-depth clinical interview, as well as providing self-reported measures of current symptom levels, additional lifetime trauma exposure, and other relevant sociodemographic information. All participants also had exposure to the same primary (index) trauma (the World Trade Center disaster in 2001). Participants reported a range of current PTSD symptom levels, from minimal symptoms (resilient) to severe cases (mean PCL-5 total score 31 19 ) - however it should be noted that due to the length of time passed since the index trauma, this represented a chronic disease course for all symptomatic individuals. Although $N=56$ is a relatively modest sample size for 
a behavioural study, simulation-based power analysis for the symptoms and task network model revealed satisfactory specificity for a discovery analysis (0.77) - minimising the chance of identifying false positive connections (estimated sensitivity of our analysis, or rate of discovery of true positives was slightly lower at 0.69: therefore, some true connections may be missing from the identified network structure).

Considering model parsimony (only two free parameters) and the continuous nature of the response variable, the latent cause model provided a generally good account of participants' loss expectancy data - however the extent to which this was true varied across subjects (Figure S2). Bivariate associations between model output and behavioural and clinical measures were therefore weighted by how informed estimates of the model parameter governing causal clustering were by task data (i.e., peakiness of the posterior probability density function for alpha values). In weighted models, likelihood of a multi-cause model $(\log B F[a l p h a>0])$ was negatively associated with extinction resistance (residual CS+ loss expectancy ratings) at the end of the task (Figure 2). LogBF values were also strikingly negatively related to failure of safety learning (loss expectancy ratings for CS- stimuli, in the absence of any association with the loss outcome) at all stages of the task (Figure S7). Individuals with lower $\log \mathrm{BF}$ values were also slower to enter ratings across the modelled period which may indicate greater uncertainty about expected values (Hyman, 1953; McDougle \& Collins, 2020).

We interpret these results as suggesting that failures of extinction learning in PTSD may relate to a primary deficit in extinction memory formation (associated with a tendency towards causal 'overgeneralization'), rather than failure to retrieve a successfully formed extinction memory (associated with a tendency towards causal hyper-segmentation). Specifically, in individuals with trauma-related psychopathology, reduced discrimination between CS + and CS- during initial learning may result in a tendency to classify multiple different potential combinations of observations (CS+, CS-, US and US omission) as being produced by the same underlying cause (Figure 2b). This may result in greater uncertainty about the likelihood of specific stimulus-outcome associations within the overarching causal structure: hampering both initial learning of correct CS-US associations (successful discriminative learning during conditioning), and subsequent learning that these associations have changed (during extinction). This explanation is consistent with the behavioural pattern observed in individuals whose behaviour supported lower likelihood of a multicause model: who showed both slower learning and less differentiated responses to CS+vs CSstimuli (Figure 2d).

Intriguingly, two recent computational studies have identified greater weighting of previous error signals during value updating for aversively-conditioned stimuli in PTSD, associated with greater volatility in stimulus value estimates (Brown et al., 2018; Homan et al., 2019). This over-correction in the face of errors in prediction might be expected if individuals with PTSD have less confidence or certainty in their internal model of the environment. Here, we propose that this results not from greater uncertainty about which causes are active in their environment, but from greater uncertainty about specific stimulus-value associations within their internal representation of that cause, exacerbated by or reflected in - a tendency to group all observations as resulting from a single underlying cause (a greater diversity of observations attributed to the same latent cause results in greater estimated 
variance of the observation prototype associated with that cause, and therefore greater likelihood that further disparate observations will be assigned to that cause; Gershman \& Niv, 2012).

An alternative explanation for our findings is that $\log B F$ values and symptom measures may both related to some other relevant individual difference, such as poorer working memory - which might predict less discriminative task performance -, or a more habitual or perseverative response style which might predict continuing to enter high loss expectancy values under extinction. Although we did not find any evidence that $\log B F$ values were associated with performance scores on a battery of neurocognitive tests probing general executive function, this data was only available in a subset of individuals $(N=24)$, and should be considered in the context of evidence of executive dysfunction in PTSD (Scott et al., 2015). Further, although ratings can be considered a relatively 'pure' measure of values or beliefs, they typically exhibit more exaggerated response functions than implicit measures (such as physiological recordings) during experimental tests of fear-conditioning (Holt et al., 2014), and may be more susceptible to certain forms of response bias. For example, it is possible that the loss expectancy data collected here are sensitive to demand characteristics (participants entering responses they believe are desired by the experimenter), and that perception of these characteristics may differ between patient and healthy samples (Orne, 1962). Future work should therefore include both attentional checks (catch trials) during task performance, and explicit questions probing beliefs about stimulus value and task structure.

It is also important to stress that, in order to facilitate remote administration, the 'aversive' outcome used in this task is highly unlikely to evoke 'fear' in the same way as stimuli used in previous work (e.g., painful electric shock). Although previous experimental tasks have successfully used monetary or game points loss in place of more primary aversive outcomes to discover differences in learning related to self-reported anxiety and PTSD symptoms (e.g., Norbury et al., 2018; Brown et al., 2018; Wise \& Dolan, 2020), more evidence is needed that the outcome employed here is engaging the kind of cognitive processes relevant to processing of traumatic experience. Future studies using this framework will therefore explicitly probe aversiveness of the loss outcome to study participants, and further, attempt to increase emotional engagement with the task by using more immersive graphics and taking a more gamified approach to task presentation (see Nord et al., 2017; Wise \& Dolan, 2020, for successful examples of this approach). Another important difference between the data presented here and that from some previous investigations is that there was no significant temporal delay between the two extinction training sessions, such that we are unlikely to be probing 'recall' of associations from longer-term memory. It is therefore possible the effects described here are specific to mechanisms subserving rapid extinction learning, which may be at least partially distinct from those supporting longer-term learning (Orederu \& Schiller, 2018).

Finally, effect sizes reported here are modest - with our measure of latent cause inference explaining around $7-8 \%$ of variance in self-reported PTSD symptoms. However, these associations persisted under a conservative (regularized) analysis approach, which tends to shrink connections weights (Epskamp et al., 2018), and after controlling for multiple other psychological symptom dimensions and clinically-relevant covariates. It was striking that $\operatorname{logBF}$ values were also associated with cumulative trauma history (independently from age), as previous work suggests that lifetime trauma load is an important predictor of vulnerability for PTSD (Karam et al., 2014; Feder et al., 2016). It 
will be necessary to test if these relationships persist in future replication samples, and if sensitivity can be increased by the various improvements to task design discussed above. A further important step will be to undertake longitudinal assessments, in order to investigate both reliability of modelbased causal inference metrics, and directionality of relationships with evolving symptom dynamics. If these challenges can be overcome, this may further our understanding of the role of high-level dysfunctional beliefs in the development and maintenance of post-traumatic stress, and perhaps even give insight into how such beliefs might be better targeted by psychological therapies (Moutoussis et al., 2017).

\section{Funding Statement}

This work was supported by CDC-NIOSH U01 awards to AF (grant numbers OH011473 and OH010729), and a NARSAD Young Investigator award from the Brain and Behavior Research Foundation to AN (grant number 28604).

\section{Data availability statement}

De-identified raw data for the contextual extinction task is available at https://github.com/agnesnorbury/latent-cause-PTSD. Clinical and demographic data are not freely publicly available due to lack of permission from study participants for public data sharing at the time of original consent, but are available from the authors on request.

\section{Conflicts of Interest}

Author AF is named co-inventor on a patent application in the USA, and several issued patents outside the USA filed by the Icahn School of Medicine at Mount Sinai related to the use of ketamine for the treatment of PTSD. This intellectual property has not been licensed. All other authors have no relevant conflicts of interest to declare.

\section{Ethical standards}

The authors assert that all procedures contributing to this work comply with the ethical standards of the relevant national and institutional committees on human experimentation and with the Helsinki Declaration of 1975, as revised in 2008. 


\section{References}

American Psychiatric Association. (2013). Diagnostic and Statistical Manual of Mental Disorders (Fifth Edition). American Psychiatric Association. https://doi.org/10.1176/appi.books.9780890425596

Armour, C., Contractor, A., Shea, T., Elhai, J. D., \& Pietrzak, R. H. (2016). Factor Structure of the PTSD Checklist for DSM-5: Relationships Among Symptom Clusters, Anger, and Impulsivity. The Journal of Nervous and Mental Disease, 204(2), 108-115. https://doi.org/10.1097/NMD.0000000000000430

Armour, C., Fried, E. I., Deserno, M. K., Tsai, J., \& Pietrzak, R. H. (2017). A network analysis of DSM-5 posttraumatic stress disorder symptoms and correlates in U.S. military veterans. Journal of Anxiety Disorders, 45, 49-59. https://doi.org/10.1016/j.janxdis.2016.11.008

Armour, C., Tsai, J., Durham, T. A., Charak, R., Biehn, T. L., Elhai, J. D., \& Pietrzak, R. H. (2015). Dimensional structure of DSM-5 posttraumatic stress symptoms: Support for a hybrid Anhedonia and Externalizing Behaviors model. Journal of Psychiatric Research, 61, 106-113. https://doi.org/10.1016/j.jpsychires.2014.10.012

Arnaudova, I., Kindt, M., Fanselow, M., \& Beckers, T. (2017). Pathways towards the proliferation of avoidance in anxiety and implications for treatment. Behaviour Research and Therapy, 96, 3-13. https://doi.org/10.1016/j.brat.2017.04.004

Badour, C. L., Blonigen, D. M., Boden, M. T., Feldner, M. T., \& Bonn-Miller, M. O. (2012). A longitudinal test of the bi-directional relations between avoidance coping and PTSD severity during and after PTSD treatment. Behaviour Research and Therapy, 50(10), 610-616. https://doi.org/10.1016/j.brat.2012.06.006

Beck, A. T., Steer, R. A., \& Brown, G. K. (1996). Manual for the Beck Depression Inventory-II. Psychological Corporation.

Békés, V., Beaulieu-Prévost, D., Guay, S., Belleville, G., \& Marchand, A. (2019). Trauma-Related Negative Cognitions Mediate the Relationship Between Avoidant Personality Beliefs and Impeded Response to Psychotherapy for PTSD. Journal of Aggression, Maltreatment \& Trauma, 28(3), 297-312. https://doi.org/10.1080/10926771.2018.1500504

Borsboom, D. (2017). A network theory of mental disorders. World Psychiatry, 16(1), 5-13. https://doi.org/10.1002/wps.20375

Bringmann, L. F., Lemmens, L. H. J. M., Huibers, M. J. H., Borsboom, D., \& Tuerlinckx, F. (2015). Revealing the dynamic network structure of the Beck Depression Inventory-II. Psychological Medicine, 45(4), 747-757. https://doi.org/10.1017/S0033291714001809

Brown, V. M., Zhu, L., Wang, J. M., Frueh, B. C., King-Casas, B., \& Chiu, P. H. (2018). Associability-modulated loss learning is increased in posttraumatic stress disorder. ELife, 7, e30150. https://doi.org/10.7554/eLife.30150

Burger, J., Isvoranu, A.-M., Lunansky, G., Haslbeck, J., Epskamp, S., Hoekstra, R. H. A., Fried, E. I., Borsboom, D., \& Blanken, T. (2020). Reporting Standards for Psychological Network. Analyses in Cross-sectional Data. PsyArXiv. https://doi.org/10.31234/osf.io/4y9nz

Contractor, A. A., Roley-Roberts, M. E., Lagdon, S., \& Armour, C. (2017). Heterogeneity in patterns of DSM-5 posttraumatic stress disorder and depression symptoms: Latent profile analyses. Journal of Affective Disorders, 212, 17-24. https://doi.org/10.1016/j.jad.2017.01.029 
de Haan, A., Landolt, M. A., Fried, E. I., Kleinke, K., Alisic, E., Bryant, R., Salmon, K., Chen, S.-H., Liu, S.-T., Dalgleish, T., McKinnon, A., Alberici, A., Claxton, J., Diehle, J., Lindauer, R., Roos, C. de, Halligan, S. L., Hiller, R., Kristensen, C. H., ... Meiser-Stedman, R. (2020). Dysfunctional posttraumatic cognitions, posttraumatic stress and depression in children and adolescents exposed to trauma: A network analysis. Journal of Child Psychology and Psychiatry, 61(1), 77-87. https://doi.org/10.1111/jcpp.13101

Duits, P., Cath, D. C., Lissek, S., Hox, J. J., Hamm, A. O., Engelhard, I. M., van den Hout, M. A., \& Baas, J. M. P. (2015). Updated Meta-Analysis of Classical Fear Conditioning in the Anxiety Disorders. Depression and Anxiety, 32(4), 239-253. https://doi.org/10.1002/da.22353

Dunsmoor, J. E., Niv, Y., Daw, N., \& Phelps, E. A. (2015). Rethinking Extinction. Neuron, 88(1), 47-63. https://doi.org/10.1016/j.neuron.2015.09.028

Epskamp, S. (2018). Preliminary simulations on the interpretation of cross-sectional Gaussian graphical models. Psy ArXiv. https://doi.org/10.31234/osf.io/54xrs

Epskamp, S., Borsboom, D., \& Fried, E. I. (2018). Estimating psychological networks and their accuracy: A tutorial paper. Behavior Research Methods, 50(1), 195-212. https://doi.org/10.3758/s13428-017-0862-1

Epskamp, S., Cramer, A. O. J., Waldorp, L. J., Schmittmann, V. D., \& Borsboom, D. (2012). qgraph: Network Visualizations of Relationships in Psychometric Data. Journal of Statistical Software, 048(i04). https://econpapers.repec.org/article/jssjstsof/v_3a048_3ai04.htm

Epskamp, S., \& Fried, E. I. (2018). A tutorial on regularized partial correlation networks. Psychological Methods, 23(4), 617-634. https://doi.org/10.1037/met0000167

Feder, A., Mota, N., Salim, R., Rodriguez, J., Singh, R., Schaffer, J., Schechter, C. B., Cancelmo, L. M., Bromet, E. J., Katz, C. L., Reissman, D. B., Ozbay, F., Kotov, R., Crane, M., Harrison, D. J., Herbert, R., Levin, S. M., Luft, B. J., Moline, J. M., .. Pietrzak, R. H. (2016). Risk, coping and PTSD symptom trajectories in World Trade Center responders. Journal of Psychiatric Research, 82, 68-79. https://doi.org/10.1016/j.jpsychires.2016.07.003

Fritz, J., Fried, E. I., Goodyer, I. M., Wilkinson, P. O., \& van Harmelen, A.-L. (2018). A Network Model of Resilience Factors for Adolescents with and without Exposure to Childhood Adversity. Scientific Reports, 8(1), 15774. https://doi.org/10.1038/s41598-018-34130-2

Gershman, S. J., Blei, D. M., \& Niv, Y. (2010). Context, learning, and extinction. Psychological Review, 117(1), 197-209. https://doi.org/10.1037/a0017808

Gershman, S. J., \& Hartley, C. A. (2015). Individual differences in learning predict the return of fear. Learning \& Behavior, 43(3), 243-250. https:/ / doi.org/10.3758/s13420-015-0176-z

Gershman, S. J., Monfils, M.-H., Norman, K. A., \& Niv, Y. (2017). The computational nature of memory modification. ELife, 6, e23763. https://doi.org/10.7554/eLife.23763

Gershman, S. J., \& Niv, Y. (2010). Learning latent structure: Carving nature at its joints. Current Opinion in Neurobiology, 20(2), 251-256. https://doi.org/10.1016/j.conb.2010.02.008

Gershman, S. J., \& Niv, Y. (2012). Exploring a latent cause theory of classical conditioning. Learning \& Behavior, 40(3), 255-268. https://doi.org/10.3758/s13420-012-0080-8

Gershman, S. J., Norman, K. A., \& Niv, Y. (2015). Discovering latent causes in reinforcement learning. Current Opinion in Behavioral Sciences, 5, 43-50. https://doi.org/10.1016/j.cobeha.2015.07.007 
Greene, T., Gelkopf, M., Epskamp, S., \& Fried, E. (2018). Dynamic networks of PTSD symptoms during conflict. Psychological Medicine, 48(14), 2409-2417. https://doi.org/10.1017/S0033291718000351

Hoffart, A., Langkaas, T. F., Øktedalen, T., \& Johnson, S. U. (2019). The temporal dynamics of symptoms during exposure therapies of PTSD: A network approach. European Journal of Psychotraumatology, 10(1), 1618134. https://doi.org/10.1080/20008198.2019.1618134

Holt, D. J., Boeke, E. A., Wolthusen, R. P. F., Nasr, S., Milad, M. R., \& Tootell, R. B. H. (2014). A parametric study of fear generalization to faces and non-face objects: Relationship to discrimination thresholds. Frontiers in Human Neuroscience, 8, 624. https://doi.org/10.3389/fnhum.2014.00624

Homan, P., Levy, I., Feltham, E., Gordon, C., Hu, J., Li, J., Pietrzak, R. H., Southwick, S., Krystal, J. H., Harpaz-Rotem, I., \& Schiller, D. (2019). Neural computations of threat in the aftermath of combat trauma. Nature Neuroscience, 22(3), 470. https:/ / doi.org/10.1038/s41593-0180315-x

Horn, S. R., Pietrzak, R. H., Schechter, C., Bromet, E. J., Katz, C. L., Reissman, D. B., Kotov, R., Crane, M., Harrison, D. J., Herbert, R., Luft, B. J., Moline, J. M., Stellman, J. M., Udasin, I. G., Landrigan, P. J., Zvolensky, M. J., Southwick, S. M., \& Feder, A. (2016). Latent typologies of posttraumatic stress disorder in World Trade Center responders. Journal of Psychiatric Research, 83, 151-159. https://doi.org/10.1016/j.jpsychires.2016.08.018

Hyman, R. (1953). Stimulus information as a determinant of reaction time. Journal of Experimental Psychology, 45(3), 188-196. https://doi.org/10.1037/h0056940

Kaczkurkin, A. N., Burton, P. C., Chazin, S. M., Manbeck, A. B., Espensen-Sturges, T., Cooper, S. E., Sponheim, S. R., \& Lissek, S. (2016). Neural Substrates of Overgeneralized Conditioned Fear in PTSD. American Journal of Psychiatry, 174(2), 125-134. https://doi.org/10.1176/appi.ajp.2016.15121549

Karam, E. G., Friedman, M. J., Hill, E. D., Kessler, R. C., McLaughlin, K. A., Petukhova, M., Sampson, L., Shahly, V., Angermeyer, M. C., Bromet, E. J., Girolamo, G. de, Graaf, R. de, Demyttenaere, K., Ferry, F., Florescu, S. E., Haro, J. M., He, Y., Karam, A. N., Kawakami, N., ... Koenen, K. C. (2014). Cumulative Traumas and Risk Thresholds: 12-Month PTSD in the World Mental Health (WMH) Surveys. Depression and Anxiety, 31(2), 130-142. https://doi.org/10.1002/da.22169

Kass, R. E., \& Raftery, A. E. (1995). Bayes Factors. Journal of the American Statistical Association, 90(430), 773-795. https://doi.org/10.1080/01621459.1995.10476572

Levy, I., \& Schiller, D. (2021). Neural Computations of Threat. Trends in Cognitive Sciences, 25(2), 151171. https://doi.org/10.1016/j.tics.2020.11.007

Lissek, S., \& van Meurs, B. (2015). Learning models of PTSD: Theoretical accounts and psychobiological evidence. International Journal of Psychophysiology, 98(3, Part 2), 594-605. https://doi.org/10.1016/j.ijpsycho.2014.11.006

Marin, M.-F., Hammoud, M. Z., Klumpp, H., Simon, N. M., \& Milad, M. R. (2020). Multimodal Categorical and Dimensional Approaches to Understanding Threat Conditioning and Its Extinction in Individuals With Anxiety Disorders. JAMA Psychiatry, 77(6), 618-627. https://doi.org/10.1001/jamapsychiatry.2019.4833 
Maruff, P., Thomas, E., Cysique, L., Brew, B., Collie, A., Snyder, P., \& Pietrzak, R. H. (2009). Validity of the CogState Brief Battery: Relationship to Standardized Tests and Sensitivity to Cognitive Impairment in Mild Traumatic Brain Injury, Schizophrenia, and AIDS Dementia Complex. Archives of Clinical Neuropsychology, 24(2), 165-178. https://doi.org/10.1093/arclin/acp010

McDougle, S. D., \& Collins, A. G. E. (2020). Modeling the influence of working memory, reinforcement, and action uncertainty on reaction time and choice during instrumental learning. Psychonomic Bulletin \& Review. https://doi.org/10.3758/s13423-020-01774-z

Moutoussis, M., Shahar, N., Hauser, T. U., \& Dolan, R. J. (2017). Computation in Psychotherapy, or How Computational Psychiatry Can Aid Learning-Based Psychological Therapies. Computational Psychiatry, 1-21. https://doi.org/10.1162/CPSY_a_00014

Norbury, A., Robbins, T. W., \& Seymour, B. (2018). Value generalization in human avoidance learning. ELife, 7. https://doi.org/10.7554/eLife.34779

Nord, C. L., Prabhu, G., Nolte, T., Fonagy, P., Dolan, R., \& Moutoussis, M. (2017). Vigour in active avoidance. Scientific Reports, 7(1), 60. https:/ / doi.org/10.1038/s41598-017-00127-6

Orederu, T., \& Schiller, D. (2018). Fast and slow extinction pathways in defensive survival circuits. Current Opinion in Behavioral Sciences, 24, 96-103. https://doi.org/10.1016/j.cobeha.2018.06.004

Orne, M. T. (1962). On the social psychology of the psychological experiment: With particular reference to demand characteristics and their implications. American Psychologist, 17(11), 776783. https://doi.org/10.1037/h0043424

Pietrzak, R. H., el-Gabalawy, R., Tsai, J., Sareen, J., Neumeister, A., \& Southwick, S. M. (2014). Typologies of posttraumatic stress disorder in the U.S. adult population. Journal of Affective Disorders, 162, 102-106. https://doi.org/10.1016/j.jad.2014.03.024

Pittig, A., Wong, A. H. K., Glück, V. M., \& Boschet, J. M. (2020). Avoidance and its bi-directional relationship with conditioned fear: Mechanisms, moderators, and clinical implications. Behaviour Research and Therapy, 126, 103550. https://doi.org/10.1016/j.brat.2020.103550

Scott, J. C., Matt, G. E., Wrocklage, K. M., Crnich, C., Jordan, J., Southwick, S. M., Krystal, J. H., \& Schweinsburg, B. C. (2015). A quantitative meta-analysis of neurocognitive functioning in posttraumatic stress disorder. Psychological Bulletin, 141(1), 105-140. https://doi.org/10.1037/a0038039

Sheehan, D. V., Lecrubier, Y., Sheehan, K. H., Amorim, P., Janavs, J., Weiller, E., Hergueta, T., Baker, R., \& Dunbar, G. C. (1998). The Mini-International Neuropsychiatric Interview (M.I.N.I): The development and validation of a structured diagnostic psychiatric interview for DSM-IV and ICD-10. The Journal of Clinical Psychiatry, 59(Suppl 20), 22-33.

Weathers, F. W., Blake, D. D., Schnurr, P. P., Kaloupek, D. G., Marx, B. P., \& Keane, T. M. (2013). Clinician-Administered PTSD Scale for DSM-5 (CAPS-5). https://www.ptsd.va.gov/professional/assessment/adult-int/caps.asp

Weathers, F. W., Litz, B. T., Keane, T. M., Palmieri, P. A., Marx, B. P., \& Schnurr, P. P. (2013). The PTSD Checklist for DSM-5 (PCL-5). https://www.ptsd.va.gov/professional/assessment/adult-sr/ptsd-checklist.asp 
Williams, D. R., Rhemtulla, M., Wysocki, A. C., \& Rast, P. (2019). On Nonregularized Estimation of Psychological Networks. Multivariate Behavioral Research, 54(5), 719-750.

https://doi.org/10.1080/00273171.2019.1575716

Williams, M. B., Karg, R. S., \& Spitzer, R. L. (2015). Structured Clinical Interview for DSM-5-Research Version (SCID-5 for DSM-5, Research Version; SCID-5-RV). American Psychiatric Association.

Wise, T., \& Dolan, R. J. (2020). Associations between aversive learning processes and transdiagnostic psychiatric symptoms in a general population sample. Nature Communications, 11(1), 4179. https://doi.org/10.1038/s41467-020-17977-w 


\begin{tabular}{lc}
\hline Age & $53(6.9)$ \\
\hline Gender (N female) & $19(34 \%)$ \\
\hline Race $(N)$ & \\
Black or African American & $6(11 \%)$ \\
Asian & $4(7 \%)$ \\
Native American & $1(2 \%)$ \\
White or Caucasian & $37(66 \%)$ \\
Other & $2(4 \%)$ \\
\hline Ethnicity $(N)$ & \\
Hispanic/Latinx & $12(21 \%)$ \\
\hline Education level (N) & $5(9 \%)$ \\
Graduated high school (or equivalent) & $17(30 \%)$ \\
Part college & $5(9 \%)$ \\
Graduated 2-year college & $14(25 \%)$ \\
Graduated 4-year college & $15(27 \%)$ \\
Graduate or professional school & \\
\hline Profession on 11/09/2001 (N) & $23(41 \%)$ \\
Traditional emergency services responder & $33(59 \%)$ \\
Non-traditional responder or survivor & $30.7(19.4)$ \\
\hline PCL-5 total score & $10.9(10.3)$ \\
\hline BDI-II total score & $3(6 \%)$ \\
\hline Psychoactive medication $(N)$ & $3(6 \%)$ \\
SSRI/SNRI (stable dose) & $3(6 \%)$ \\
NDRI (stable dose) & $16(29 \%)$ \\
sedative (night-time use only) & $7(13 \%)$ \\
\hline Additional lifetime trauma history & \\
N trauma categories endorsed $(0-13)$ & \\
Childhood physical abuse $(N)$ & \\
Childhood sexual abuse $(N)$ & \\
Adulthood sexual trauma $(N)$ & \\
\hline & \\
& \\
\hline
\end{tabular}

01/02/2021 14:55:00Table 1. Summary of demographic and clinical variables for study participants ( $N=56)$. Values represent mean (SD) unless otherwise specified. Race/ethnicity and medication status categories are non mutually-exclusive; $N=8(14 \%)$ individual participants were currently taking a stable dose ( $>3$ months) of a psychoactive medication. PCL-5, PTSD Checklist for DSM-5; BDI-II, Beck Depression Inventory version II; SSRI, selective serotonin reuptake inhibitor; SNRI, serotonin/noradrenaline reuptake inhibitor; NDRI, noradrenaline/dopamine reuptake inhibitor. For further information on PTSD and depression subscore ranges and distributions, see Table S1. All study participants had DSM-5 Category A trauma exposure to the World Trade Center disaster in 2001. For details about additional lifetime trauma categories, and how these were defined, see Table S2. 


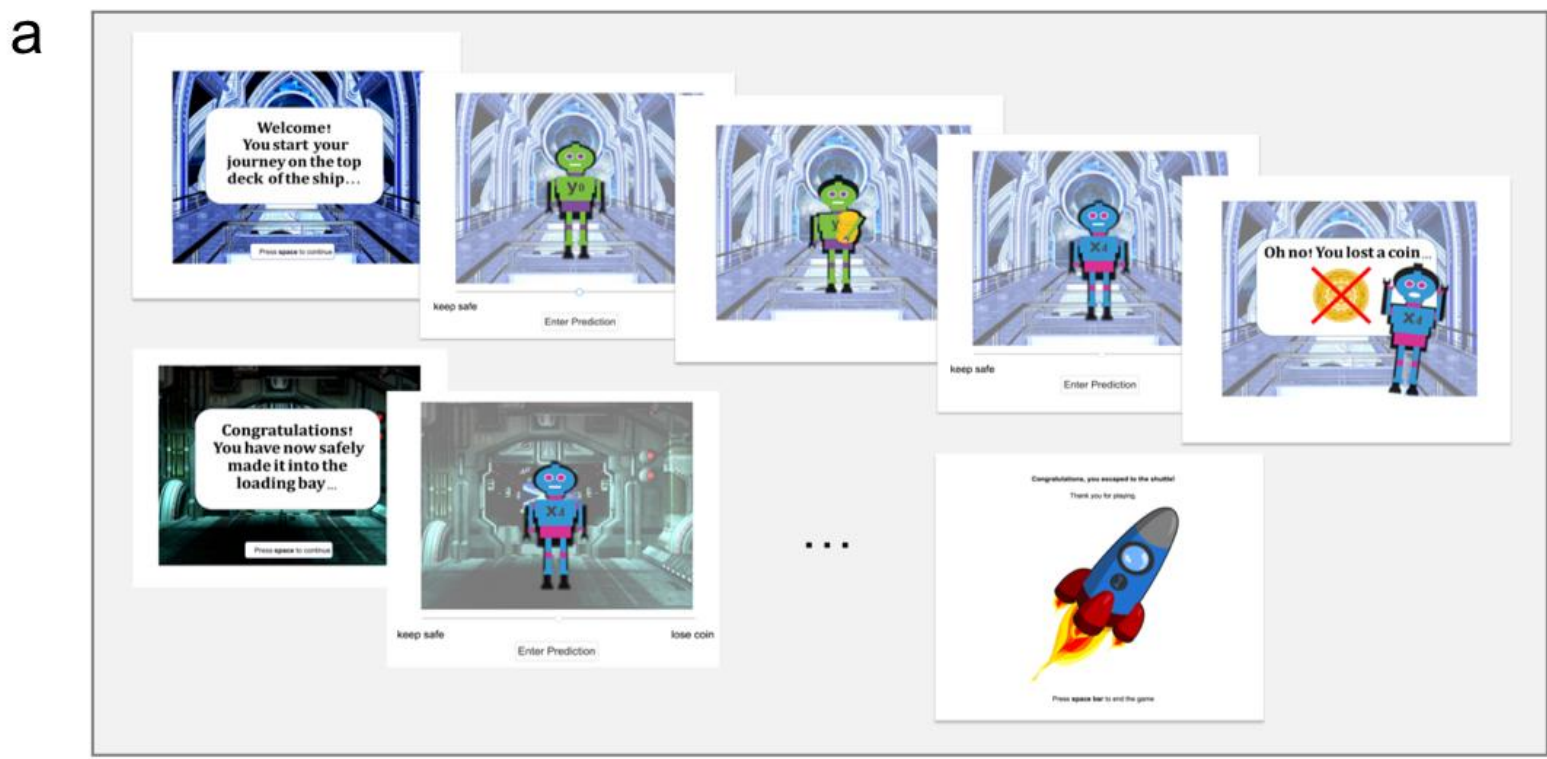

b

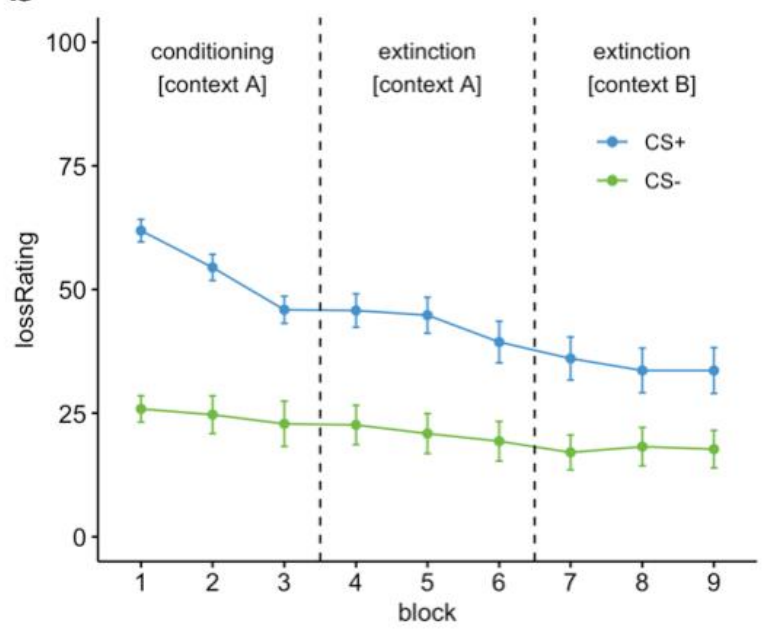

C

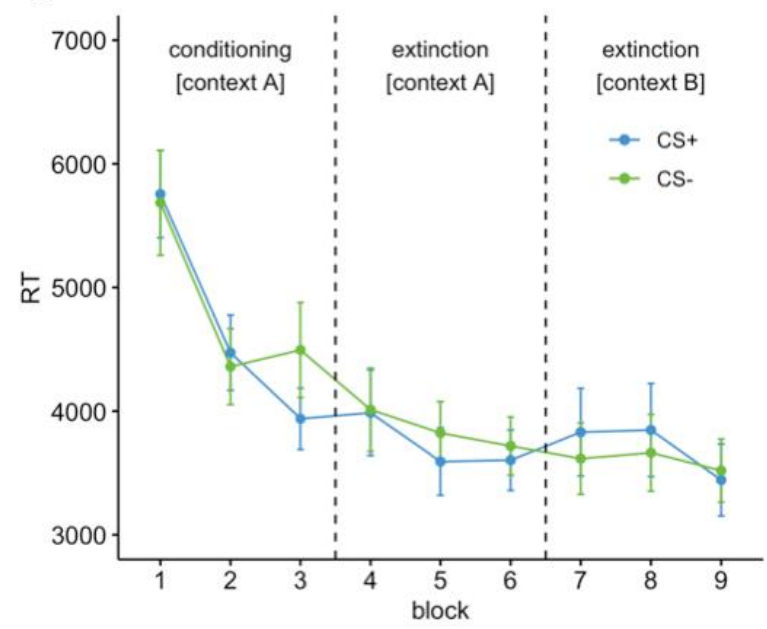

Figure 1. Data from the online extinction learning task demonstrated that participants learned to discriminate between conditioned and unconditioned stimuli, and to decrease loss expectancy ratings for conditioned stimuli following the transition to extinction. a Depiction of trials from the online extinction learning task. Participants were told that they were travelling through different zones of a spaceship, and needed to escape with enough space coins to power their journey home. Unfortunately, the coins needed to be carried by helper robots, some of whom were unreliable. On each trial, participants encountered a robot and rated how likely they think that robot would be to lose one of their coins using a sliding bar (participants were informed that their ratings would not change the outcome they observed, but that their predictions should be as accurate as possible in order to aid future space travellers). $P($ lose a coin $\mid \mathrm{CS}+$ ) was $1 / 3$ during initial conditioning, and reduced to 0 during extinction training stages, ( $($ (lose a coin|CS-) was always 0$)$. The transition between conditioning and initial extinction learning stages was unsignalled, but the final stage of the task (further extinction training in a novel context B) occurred following transition to a different 'zone' of the ship (signalled by a change of background image). $\mathbf{b}$ Mean loss expectancy ratings across participants, by CS type and task stage (three blocks with ten trials per block). c Median response times (RTs) to input ratings, by CS type and task stage. Error bars represent standard error of the mean. CS+, aversively conditioned (loss-associated) stimulus; CS-, non loss-associated stimulus. 
a

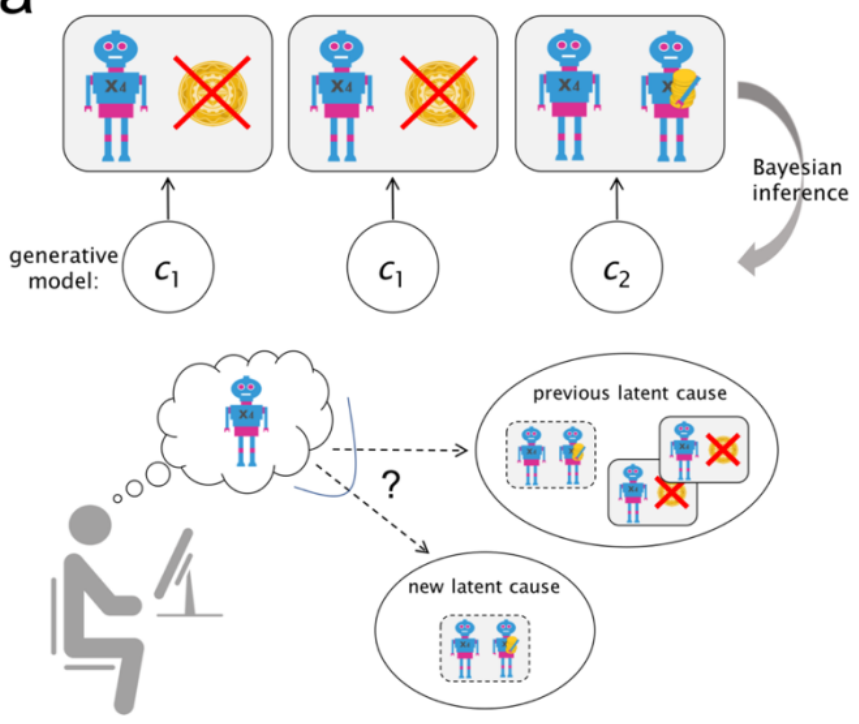

$P($ cause $=c \mid$ stimuli $) \propto P($ stimuli $\mid$ cause $=c) * P($ cause $=c)$

inferred probability of consistency between current prior bias towards greater cause $c$ being active on stimuli and those predicted for or fewer causes, governed this trial
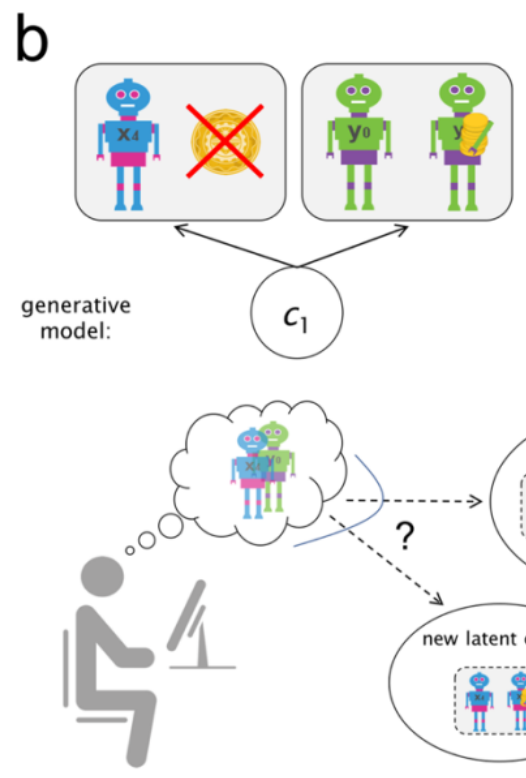

by $a$

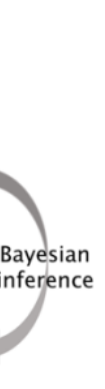

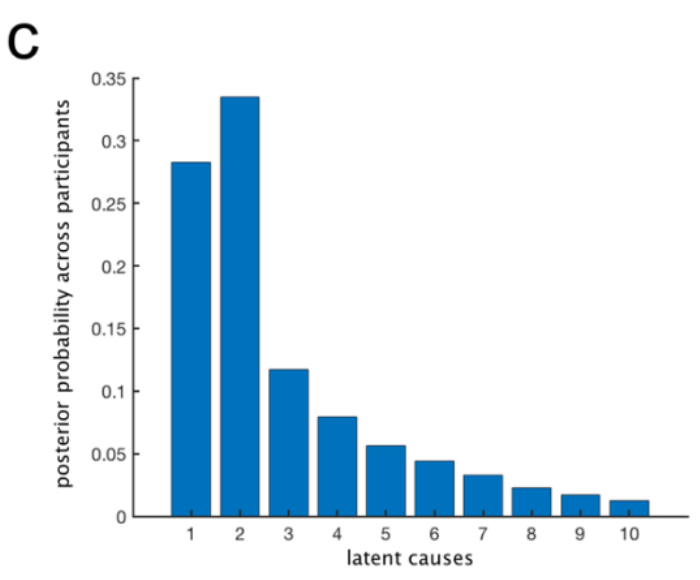
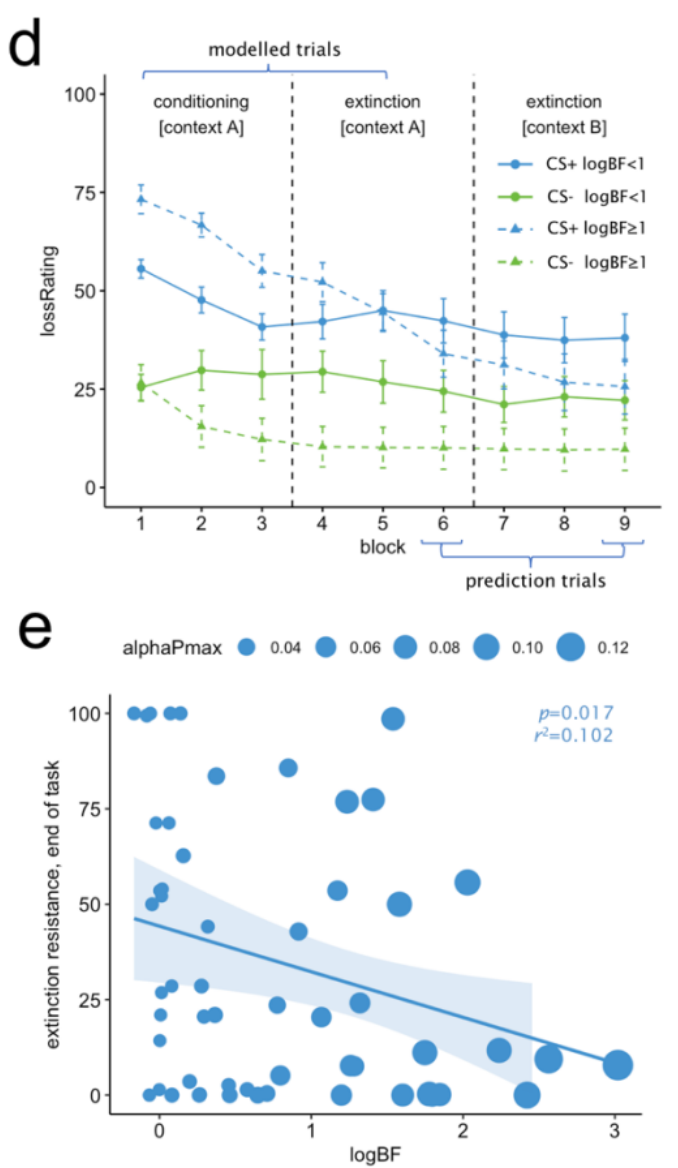

Figure 2. Latent cause modelling of extinction task data revealed that trauma-exposed individuals whose extinction task data were better explained by a single cause model discriminated less between conditioned and unconditioned stimuli during initial learning, and showed greater resistance to extinction. a The model posits that during learning an individual attempts to infer which latent cause is responsible for their observations, based on their previous experience of the task and prior beliefs about causal structure of the environment. On each trial, individuals may infer that a previous cause is responsible for their observations, or that something in the underlying task structure has changed, and observations should be assigned to a new cause. The probability of assigning an observation to a new cause is proportional to the dissimilarity between the current stimuli and those predicted for the current cause, and individual preference towards simpler or more complex causal structures (governed by a single parameter, $\alpha$ ). According to one account, individuals with fear-learning disorders may be more 
likely to assign extinction trial observations to a new underlying cause, rendering them susceptible to extinction relapse (spontaneous return of fear) if, for some reason, they infer that the original cause is active again (e.g., when times passes or contextual cues change). $\mathbf{b}$ Under an alternative account, individuals with fear-learning disorders may have a fundamental deficit in distinguishing trials involving aversively conditioned (CS+) and unconditioned (CS-) stimuli (e.g., due to over-generalization of aversive information, or hampering of safety learning by hyperarousal). Disparate configurations of stimuli and outcomes (CS+, CS-, US, and US omission) may be clustered together in the inferred causal structure of the environment, leading to greater uncertainty about the pattern of stimuli and outcomes associated with a given cause, and therefore slower learning of expected values during both initial conditioning and later extinction stages. $\mathbf{c}$ The marginal probability distribution of latent causes averaged across all participants indicated that most participants inferred that one or two causes were responsible for their observations across conditioning and extinction stages (other causes had relatively low posterior probabilities). $\mathbf{d}$ The likelihood that an individual's internal model of the task contained more than one cause can be quantified as the log Bayes' Factor $(\log \mathrm{BF}$ ) for a model where $\alpha>0$, compared to single cause model (where $\alpha=0$ ). For illustration, behavioural data is displayed separately for individuals for whom model comparison favoured a model with more than one cause ( $\log B F \geq 1$, dotted lines, $N=20$ ), and individuals for whom model comparison found no strong evidence for a multicause model $(\log B F<1$, solid lines, $N=36)$. The latter group tended to learn more slowly across the task (flatter curves) and showed less discrimination in loss expectancy ratings between CS+ and CS- stimuli. Error bars represent standard error of the mean. e Lower $\log B \mathrm{~F}$ values (calculated from conditioning and extinction stage data only) were associated with higher resistance to extinction scores (residual CS+ loss expectancy ratings) at the end of the task. Regression line and $p$ value represents linear model fit, weighted by posterior certainty in $\alpha$ parameter estimates (alphaPmax; higher certainty $=$ larger dot size). Panels $a$ and $b$ are adapted from Gershman et al., (2015). 

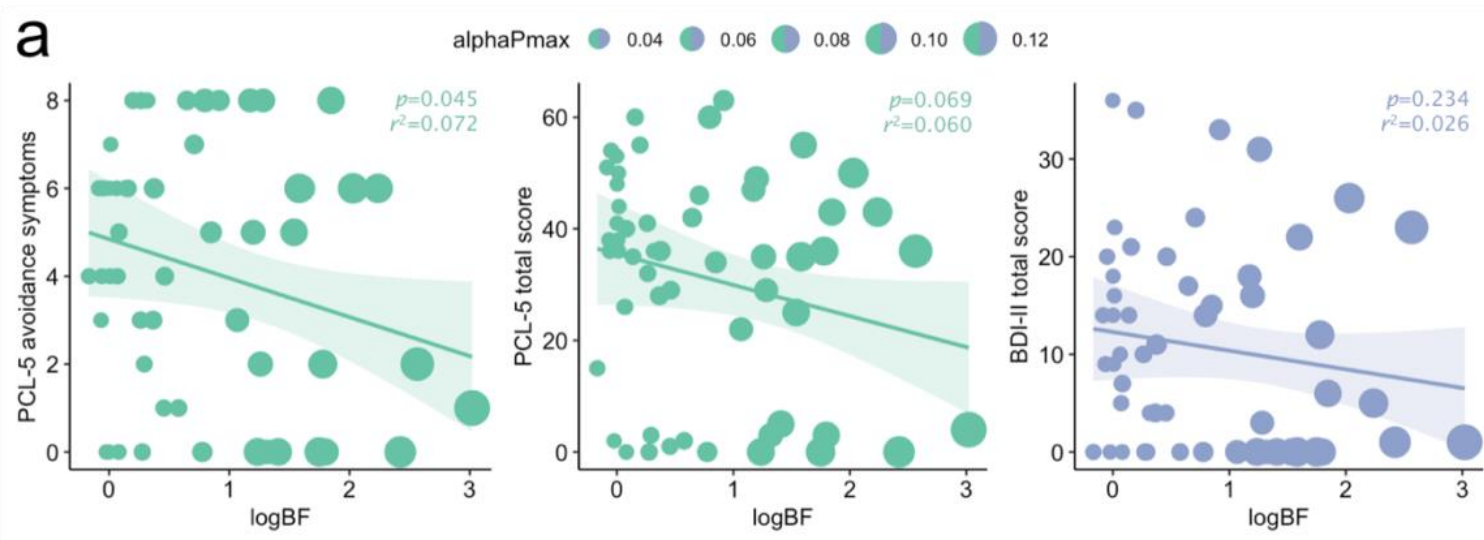

b

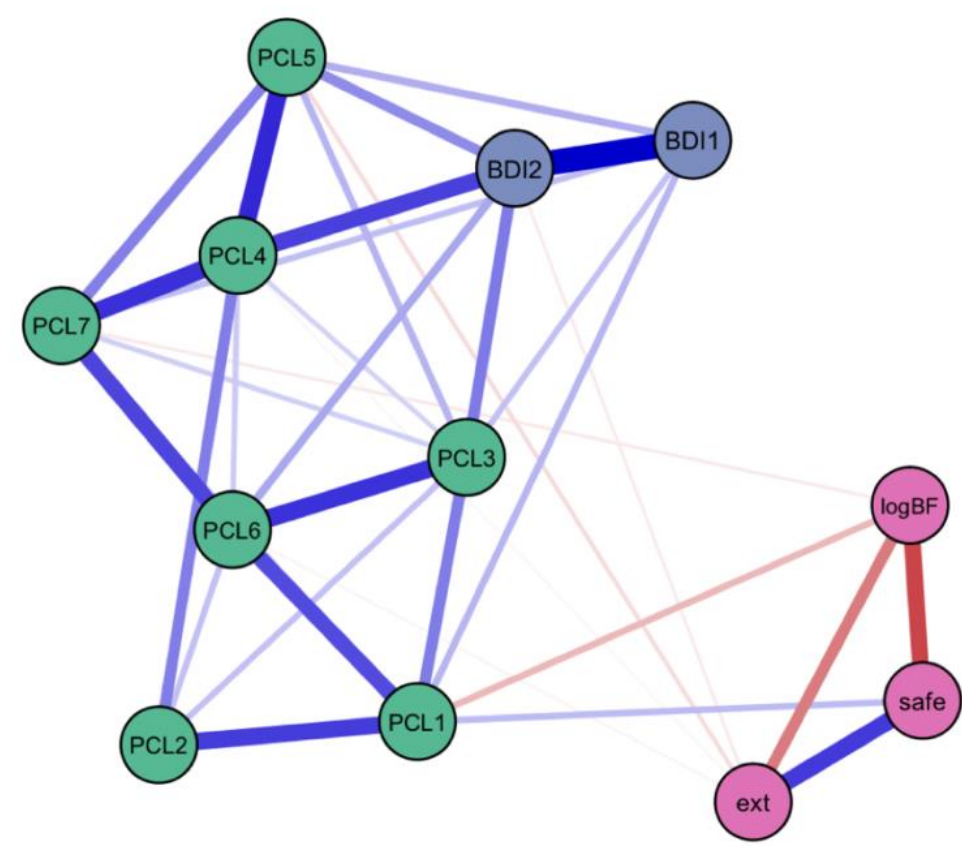

PTSD symptoms

- PCL1: re-experiencing

- PCL2: avoidance

- PCL3: negative affect

- PCL4: anhedonia

- PCL5: externalizing behaviour

- PCL6: anxious arousal

- PCL7: dysphoric arousal

Depression symptoms

- BDI1: cognitive symptoms

- BDI2: physical/affective symptoms

\section{Extinction task}

- $\log B F: \log B F($ alpha $>0)$

- ext: extinction resistance

- safe: safety learning failure

C

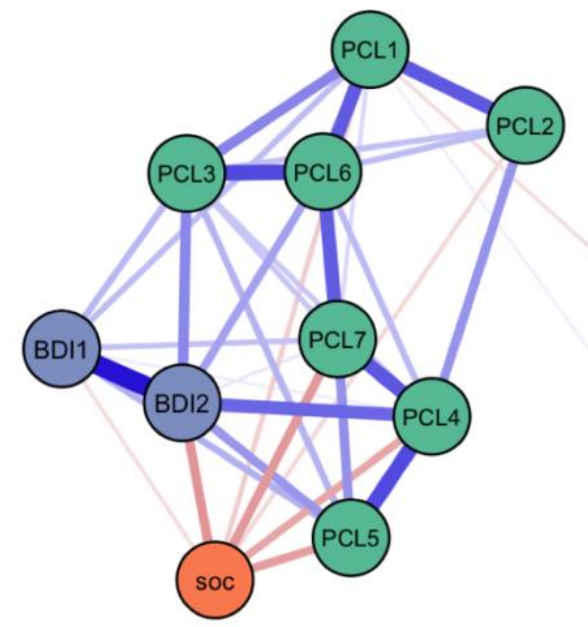

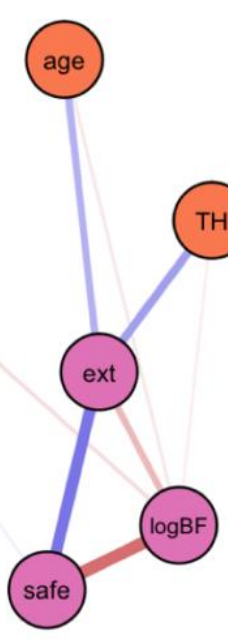

$\mathrm{TH}$

H PCL5: externalizing behaviour

- PCL6: anxious arousal

- PCL7: dysphoric arousal

Depression symptoms

- BDI1: cognitive symptoms

- BDI2: physical/affective symptoms

\section{Extinction task}

- $\log B F: \log B F(a l p h a>0)$

- ext: extinction resistance

- safe: safety learning failure

\section{Clinical covariates}

$\circ$ age: age

- edu: education level

- soc: perceived social support

- TH: additional (nonWTC) trauma 
Figure 3. Trauma-exposed individuals with greater tendency to infer a single cause was responsible for observations across conditioning and extinction stages reported more severe PTSD, but not depression, symptoms. a Bivariate relationships between latent cause inference and avoidance, total PTSD, and total depression symptoms. $\log$ BF represents log Bayes' Factor for a model with more than one cause $(\alpha>0)$, compared to a single cause model $(\alpha=0)$. PCL-5, PTSD Checklist for DSM-5; BDI-II, Beck Depression Inventory, version II. $p$ values represent the results of linear regression models, weighted by posterior certainty in the value of $\alpha$ (alphaPmax; higher certainty=larger dot size). b Regularized network model incorporating clinical symptom dimensions (seven PCL-5 PTSD and two BDI-II depression symptom dimensions) and extinction task performance measures (extinction resistance, or mean residual CS+ loss expectancy at the end of the task; safety learning failure, or mean CS- loss expectancy at the end of the task; and $\log \mathrm{BF}$, indexing latent cause inference across initial conditioning and extinction learning trials). c Regularized network model incorporating PTSD and depression symptoms, extinction task performance measures, and clinically-relevant covariates: specifically age, self-reported education level, perceived social support (MOS-SS total score), and additional lifetime trauma history. For both networks, connections between nodes (edges) represent partial correlation coefficients retained following LASSO regularization, a conservative approach which favours a sparse network structure and removes spurious edges. Blue edges represent positive connections, and red edges negative connections. Greater line width and stronger colour intensity represent greater edge strength, with edge weights plotted using the same scale in order to be comparable across networks (max value $=0.4)$. 
Supplementary Material 


\section{Supplemental Methods}

\section{Participants}

Inclusion/exclusion criteria. Participants fell into two broad categories: individuals who currently met diagnostic criteria for full or subthreshold PTSD, and individuals who were assessed as never having met criteria for PTSD or any other DSM-5 Axis-1 disorder. As per previous studies from our research group (e.g., Chen et al., 2020), subthreshold PTSD was defined according to two commonly-used sets of criteria, adapted for DSM-5 (see McLaughlin et al., 2015). Specifically, this required the presence of one Criterion B (intrusion) symptom, plus 1) either three symptoms from across Criteria C (avoidance) and D (negative alterations in mood or cognition), or two symptoms from Criterion $\mathrm{E}$ (alterations in arousal and physiological reactivity); or 2) one criterion $\mathrm{C}$ or $\mathrm{D}$ symptom plus one Criterion E symptom.

Participants with a lifetime history of a primary psychotic or bipolar disorder, alcohol or other substance use disorder within the prior three months, current uncontrolled medical illness, disorder of the central nervous system, or history of head injury were not recruited. Participants were further excluded if they were currently taking antipsychotic, mood stabilising, or opioid medications, or reported day-time use of sedative medications. Participants taking other psychoactive medications (e.g. SSRIs) were required to be on a stable dose (same medication and dose for at least three months, prior to taking part). All data analysed here were collected between 06/03/19 and 23/02/20, i.e., prior to the onset of the Covid-19 pandemic in the USA.

Trauma history. $N=25$ participants provided information about additional lifetime trauma exposure using the Traumatic Life Events Questionnaire (TLEQ; Kubany et al., 2000), and N=31 using the Trauma History Screen (THS; Carlson et al., 2011). Items probing common trauma categories across both measures were used to derive a non frequency-weighted trauma history score for the presence/absence of 13 common traumatic experiences (e.g., life-threatening illness or injury, natural disaster, sexual violence; Table S2). Although items are not completely interchangeable, THS and TLEQ scores are moderately strongly correlated $(r=0.73-77)$, and median agreement between measures by trauma category was previously found to be $72-78 \%$ in community samples of adults (Carlson et al., 2011). Total score on this measure was used for further analysis on the basis of evidence that the burden of trauma is cumulative across lifespan and this predicts likelihood of PTSD from WTC trauma (Karam et al., 2014; Feder et al., 2016).

Demographic information, social support, and cognitive function. Participants completed a demographic questionnaire where they provided information about their age, gender, race/ethnicity, and highest level of educational achievement. All participants also completed the Medical Outcomes Survey brief Social Support questionnaire (MOS-SS; Sherbourne \& Stewart, 1991), which was included in the analysis as perceived social support has previously been shown to play an important role in resilience to psychopathology following trauma (Fritz et al., 2018; Stevens \& Jovanovic, 2019). A subset of individuals $(N=24)$ completed the Cogstate battery, a set of computerised tests probing general executive function that have been shown to be sensitive to mild cognitive impairment (Maruff et al., 2009). Specifically, participants completed the identification test (attention), detection test (psychomotor function), Groton maze learning tasks (executive function and memory), international shopping list test (verbal learning and memory), one card learning test 
(visual learning), and the one-back task (working memory). Primary outcome measures for each test were combined to form composite cognitive function scores ( $z$ scored across participants), as described in the Cogstate manual.

\section{Extinction learning task}

The extinction task was administered online, via web browser. Following instructions screens outlining the cover story and images of example trials, a short true/false quiz tested understanding of the task. Participants scoring less than $3 / 3$ correct were routed back to the start of the instructions screens to try again.

On each trial, participants were presented with an image of a robot and asked to rate their expectancy of that particular losing a coin using a sliding bar with the anchors "keep safe" [0] and "lose coin" [100]. After participants input their prediction, the outcome of that trial was displayed (a simple animation representing either loss of a coin, the aversive outcome, or retention of coins, the neutral outcome). Participants were informed at the start of the task that their ratings would have no impact on the outcomes they observed, but should be made as accurately as possible, in order to aid future space travellers.

Each stage of the experiment (conditioning [in context A], initial extinction training [in context A], and further extinction training [in novel context B]) consisted of 3 blocks of 10 trials (90 trials total). CS + stimuli were reinforced on $1 / 3$ of trials in conditioning phase only: the ratio of trial types was 4:2:4 CS+(unreinforced):CS+(reinforced):CS- during conditioning, and 4:0:4 during both extinction stages. Responses were not speeded (trials did not progress until a rating had been entered), and participants had the option to take a break for as long as they liked at the end of each block of 10 trials. The identities of CS+ and CS- stimuli (defined by a combination of robot colours and markings) were counterbalanced across participants and all participants experienced the same pseudorandom trial sequence (and therefore identical outcomes).

The original purpose of including novel context B in the task design was to test for the possibility of detecting renewal effects related to contextual cue changes. However, in the analysis presented here we do not further investigate renewal or recall effects as 1) we observed differential responding to $\mathrm{CS}+$ and CS- stimuli at the end of the initial learning stage, indicating incomplete learning, and 2) the lack of temporal delay between the two extinction training phases means we are unlikely to be tapping into cognitive mechanisms underlying recall (Orederu \& Schiller, 2018).

Stimuli were generated using royalty-free vector art from the pixabay database (https://pixabay.com/), and were checked for distinctiveness under common forms of colour blindness using the Color Oracle simulator (https:// colororacle.org/). The task was programmed in javascript using tools from the jsPsych library (de Leeuw, 2015), and deployed using Pavlovia (https://pavlovia.org/).

\section{Analysis}

\section{Extinction task data}

Manipulation check. Overall effects of task on loss expectancy ratings and response time data were investigated using repeated-measures ANOVA of linear mixed-effect models with error terms 
included as random effects (lmer function from the R package lmerTest). Within-subject factors in the model were CS type (CS+ vs CS-) and task stage (conditioning, initial extinction learning in original context A, and further extinction learning in novel context B). Follow-up contrasts to explore the direction of effects used the Tukey LSD correction for multiple comparisons.

\section{Latent cause modelling of extinction task data}

Model fit procedure. As per Gershman and Hartley (2015), we used a linear observation function with single scaling parameter beta to fit model-generated $P($ loss $)$ on each trial to observed loss probability ratings. Specifically,

$$
\text { lossRating }=\beta * \sum_{c} P(\text { loss } \mid \text { cause }=c) * P(\text { cause }=c \mid \text { stimuli })+\varepsilon
$$

where $\varepsilon$ is a noise parameter drawn from a normal distribution with mean $=0$ and variance $=1$.

Values of parameters $\alpha$ and $\beta$ that maximized the likelihood of an individuals' ratings data were found using the non-linear optimization method described in (Gershman \& Niv, 2012; Gershman \& Hartley, 2015). Briefly, this is a sampling approach based on summing over sets of hypothetical state sequences or particles (particle filtering). Here, we used $M=500$ particles. Values of parameters were estimated for each participant separately (i.e., non-hierarchically). The model was fit using a uniform prior across possible stimulus configurations, such that, a priori, all possible prototypical state configurations were equally likely. The maximum number of latent causes an individual could infer over was 10 , and $\alpha$ values were allowed to range $[0,10]$ ( $N=50$ linearly spaced values were evaluated). Loss expectancy ratings data were z scored within-participants prior to analysis.

Simulation and recovery analysis. In order to assess stability of parameter estimation on our paradigm, simulation and recovery analysis was conducted. Specifically, random samples $(N=250)$ were drawn from the observed distributions of the Chinese Restaurant Process concentration parameter $\alpha$ and scaling parameter $\beta$ across participants, and used to simulate loss expectancy ratings data in response to the sequence of stimuli and outcomes experienced by study participants. The latent cause model was then fit to the simulated data (using the same procedure as described for the experimental data), and recovered parameter values regressed against the simulated values. In order to further test the reliability of the model output submitted to further analysis here (logBF, or $\log$ likelihood of a model where $\alpha>0$, compared to a model were $\alpha=0)$, the randomly generated parameter samples were enriched at $\alpha=0$ ( $N=25$ additional samples), and recovered logBF scores were compared between cases where simulated $\alpha$ values were $>$ or $=$ to 0 .

\section{Network analysis}

Data pre-processing. Clinical measures exhibited skewed distributions across the sample (see histograms in Table S1), and so were transformed using the nonparanormal function (huge.npn from the R package huge; Zhao et al., 2012) prior to network estimation. All networks reported here are based on npn-transformed Pearson correlations.

Network estimation. Network estimation followed previously published procedures (Epskamp \& Fried, 2018; Fried \& Burger, 2019). Networks were constructed using regularized Gaussian graphical estimation implemented in the R package qgraph, version 1.6.5 (Epskamp et al., 2012). In order to deal with the relatively small number of observations (participants) compared to potential network 
connections, least absolute shrinkage and selection operator (LASSO) regularization (Tibshirani, 1996) was applied to the underlying partial correlation matrix. This form of regularization causes weak edge estimates to shrink to zero, resulting in a sparse, conservative network structure (Friedman et al., 2008). The LASSO tuning parameter governing degree of regularization was chosen via model selection based on the extended Bayesian Information Criterion (EBIC; Foygel \& Drton, 2010), with EBIC hyperparameter gamma set to the default value (0.5).

Estimation stability and power assessment. Stability of network parameter estimation was assessed using the R package bootnet, version 1.4.3 (Epskamp et al., 2018). Specifically, edge weight accuracy was assessed using nonparametric bootstrapping, whereby observations in the data were resampled with replacement to create 2000 plausible new datasets. These bootstrapped estimates were then used to estimate the sampling distribution of network edge weights, and therefore construct $95 \%$ confidence intervals (CIs), such that in $95 \%$ of the cases the CI will contain the true value of the parameter. $N B$, these CIs are not intended to be used to draw inferences about the significance of an edge in the network (distributions of LASSO-regularized parameters are substantially non-normal therefore approximate $p$-values are not easy to obtain via bootstrap sampling). The presence of an edge in a LASSO-regularized model implies that it is sufficiently strong to be included in the model. However, a wide CI means that it may be hard to interpret the strength of that edge (Epskamp et al., 2018). Due to our relatively small $N$ (and likely instability of exact edge strength estimates), we do not report differences in edge strength between nodes, or node centrality indices.

Power of the network analysis was assessed using the netSimulator function from bootnet, as per Faelens et al., (2019). Specifically, we used the discovered ('true') network structure to simulate 1000 networks at various sample sizes $(N=56,100,150,500)$, and then examined how well the simulated networks recovered the true network structure. This was assessed using three metrics: correlation between the simulated and true networks, sensitivity (rate of discovery of network connections present in the true network), and specificity (rate of discovery of network connections absent in the true network).

Visualisation. Networks are plotted with layout determined by the Fruchterman Reingold algorithm (qgraph argument "spring"; Fruchterman \& Reingold, 1991): a force-directed graph method which positions nodes so as to minimize crossing edges (Jones et al., 2018). Edges are plotted according to the colourblind-friendly scheme included with qgraph: with positive connections in blue, and negative connections in red. In order to aid visual comparison of networks with and without additional clinically-relevant covariates, edge weights were plotted on the same scale (maximum edge weight for each network set to the maximum value across networks). 


\section{Supplemental Results}

\section{Extinction learning task}

Manipulation check. In order to check if participants performed as expected on the task, loss expectancy ratings were analysed by repeated-measures ANOVA. This analysis revealed significant effects of CS type and task stage on mean loss prediction ratings $\left(F_{1,947}=247.1, p<2.2 \mathrm{e}-16\right.$; $F_{2,947}=27.5, p=2.5 \mathrm{e}-12$; respectively) - with higher ratings for CS + compared to unconditioned (CS-) stimuli (mean difference 22.9[SE 1.5], 95\%CI 20.1-25.8), and decreases in mean loss expectancy ratings between conditioning and extinction stages (from conditioning to initial extinction stages mean decrease was 7.1[SE 1.8] points, 95\% CI 3.0-11.3; from initial extinction and further extinction training stages mean decrease was 6.1[SE 1.8] points, 95\%CI 1.9-10.3; both differences $p<0.002$ ). There was also a significant interaction between CS type and task stage $\left(F_{2,947}=6.52, p=0.002\right)$, with a greater decrease in ratings for $\mathrm{CS}+$ stimuli $(\mathrm{CS}+$, rating decrease from conditioning to further extinction training stage of 19.7 [SE 2.5], 95\% CI 12.4-26.9, $p=2.0 \mathrm{e}-13$; CS-, rating decrease of 6.8 [SE 2.5], $95 \%$ CI $-0.4-14.0, p=0.08)$. This indicates that overall, participants entered greater loss expectancy ratings for the aversively conditioned (CS+) compared to non aversively-conditioned (CS-) stimuli, and decreased their ratings of CS+, but not CS-, stimuli over the course of the task (i.e., when these stimuli began to be presented in extinction).

There was also a significant effect of experiment stage on response times $\left(F_{2,947}=61.8, p<2.0 \mathrm{e}-16\right)$, with quicker response times at later stages in the task (mean decrease in RT from conditioning to initial extinction stages of 995ms[SE 111], 95\%CI 735-1256, $p<0.001$; difference in RT from initial to further extinction training stages, $p>0.4$ ). There was no evidence for an effect of CS type or interaction between CS type and experiment stage on response timing (both $p>0.4$ ).

\section{Latent cause modelling of extinction task data}

Looking inside the model. Since the choice of threshold on $\operatorname{logBF}$ scores used to visualise differences in task behaviour associated with differences in model output was somewhat arbitrary, we tested whether pattern reported in Figure $2 \mathrm{~d}$ was robust to different choices of threshold. Specifically, we tested if the pattern was robust to reductions in threshold from $\operatorname{logBF} \geq 1$ (chosen on the basis of representing 'strong' evidence in favour of a multi-cause model; Kass \& Raftery, 1995) to values closer to 0 (representing the turning point at which evidence begins to favour the null hypothesis; for reference, a previous investigation of latent cause inference during extinction learning in healthy participants used a threshold of 0.088 , based on minimal differences in extinction success between groups; Gershman \& Hartley, 2015). At thresholds of $\log B F \geq 0.5$ and $\log B F \geq 0.1$, there was again evidence of slower extinction learning (flatter gradients of CS + loss expectancy ratings; $p=0.004, p=0.028$, Wilcoxon signed-rank tests) and less discrimination between CS + and CS- stimuli (both $p<0.001$, Wilcoxon signed-rank tests), in the lower logBF groups (representing $N=29, N=17$ individuals, respectively; at lower thresholds there were too few participants in the low $\operatorname{logBF}$ group for meaningful comparison). Thus, the pattern of behavioural differences we observed was robust to changes in definition of this threshold (Figure S6).

Relationship to response times. Since the relationship between block number and median response times appears to be non-linear (Figure 1c), trial-by-trial associations between response 
times and estimated uncertainty over latent causes were also tested using an exponential decay function to represent the time-on-task effect, rather than a linear function (trial number). Specifically, a simple exponential decay function of the form $b^{*}\left(1 /\left(1+\operatorname{trial}^{\wedge} \mathrm{c}\right)\right)$ was fit to normalized median RTs across all subjects, and the resulting function used to generate a monotonically decreasing time-on-task regressor. In linear mixed models with either a linear or exponential time-on-task regressor, there were significant effects of time-on-task on trial RT, with quicker responses at higher trial numbers or lower values of the decay function ( $\beta=-63.0$ [SE 5.4], $\beta=6627$ [SE 453], respectively; both $p<0.001)$. In both models, responses were slower when modelled probability of the US (loss outcome) was greater ( $\beta=5758$ [SE 639], $\beta=2523$ [SE 731], respectively; both $p<0.001$ ), and when uncertainty about the specific underlying cause was lower (higher maximum probability across causes: $\beta=9457$ [SE 456]; $\beta=2811$ [SE 662], both $p<0.001$ ). In both models, lower $\log \mathrm{BF}$ values were associated with greater response times, across trials $(\beta=$ 1395[SE 456], $p<0.001 ; \beta=-847[$ SE 373], $p=0.027$ ). 


\begin{tabular}{|c|c|c|c|}
\hline Clinical variable & Nitems & Summary statistics & Histogram \\
\hline $\begin{array}{l}\text { PCL-5 } \\
\text { re-experiencing } \\
\text { symptoms }\end{array}$ & 5 & $\begin{array}{l}\operatorname{mean}(\mathrm{SD}): 7.9(5.3) \\
\min <\operatorname{med}<\max : 0<9<18 \\
\mathrm{IQR}(\mathrm{CV}): 8.5(0.7)\end{array}$ & \\
\hline $\begin{array}{l}\text { PCL-5 } \\
\text { avoidance symptoms }\end{array}$ & 2 & $\begin{array}{l}\operatorname{mean}(\mathrm{SD}): 4.1(2.8) \\
\min <\operatorname{med}<\max : 0<4<8 \\
\text { IQR }(C V): 4.2(0.7)\end{array}$ & \\
\hline $\begin{array}{l}\text { PCL-5 } \\
\text { negative affect }\end{array}$ & 4 & $\begin{array}{l}\text { mean }(\mathrm{SD}): 4.5(3.6) \\
\min <\operatorname{med}<\max : 0<4.5<12 \\
\text { IQR }(\mathrm{CV}): 6.2(0.8)\end{array}$ & \\
\hline $\begin{array}{l}\text { PCL-5 } \\
\text { anhedonia }\end{array}$ & 3 & $\begin{array}{l}\text { mean }(\mathrm{SD}): 4.5(3.8) \\
\min <\operatorname{med}<\max : 0<4.5<12 \\
\text { IQR }(\mathrm{CV}): 0.0(0.9)\end{array}$ & \\
\hline $\begin{array}{l}\text { PCL-5 } \\
\text { externalizing } \\
\text { behaviours }\end{array}$ & 2 & $\begin{array}{l}\operatorname{mean}(\mathrm{SD}): 2.2(2.2) \\
\min <\operatorname{med}<\max : 0<2<9 \\
\text { IQR }(C V): 3.0(1.0)\end{array}$ & \\
\hline $\begin{array}{l}\text { PCL-5 } \\
\text { anxious arousal }\end{array}$ & 2 & $\begin{array}{l}\operatorname{mean}(\mathrm{SD}): 3.89(2.7) \\
\min <\operatorname{med}<\max : 0<4<8 \\
\text { IQR }(C V): 4.2(0.7)\end{array}$ & \\
\hline $\begin{array}{l}\text { PCL-5 } \\
\text { dysphoric arousal }\end{array}$ & 2 & $\begin{array}{l}\text { mean }(\mathrm{SD}): 3.6(2.7) \\
\min <\operatorname{med}<\max : 0<4<8 \\
\text { IQR }(C V): 6.0(0.7)\end{array}$ & \\
\hline $\begin{array}{l}\text { BDI-II } \\
\text { cognitive symptoms }\end{array}$ & 7 & $\begin{array}{l}\text { mean }(\mathrm{SD}): 3.2(4.0) \\
\min <\operatorname{med}<\max : 0<1.5<18 \\
\text { IQR }(\mathrm{CV}): 5.2(1.2)\end{array}$ & \\
\hline $\begin{array}{l}\text { BDI-II } \\
\text { physical/affective } \\
\text { symptoms }\end{array}$ & 14 & $\begin{array}{l}\text { mean }(\mathrm{SD}): 7.75(7.0) \\
\min <\operatorname{med}<\max : 0<6<24 \\
\text { IQR }(C V): 14.0(0.9)\end{array}$ & \\
\hline
\end{tabular}

Table S1. Summary statistics and distribution of scores for PTSD and depression symptom dimensions. $\mathrm{N}$ items, number of individual items contributing to each symptom cluster. PCL-5, PTSD Checklist for DSM-5; BDI-II, Beck Depression Inventory version II; IQR, interquartile range; CV, coefficient of variation (relative standard deviation). PCL-5 PTSD symptom dimensions are defined as per Armour et al., 2015. BDI-II depression symptom dimensions are defined as per Bringmann et al., 2015. Table generated using the R package summarytools (https:// cran.r-project.org/package= summarytools). 


\begin{tabular}{l} 
THS item \\
\hline 1. Life-threatening illness or injury \\
2. A really bad car, boat, train, or airplane accident
\end{tabular}

2. A really bad car, boat, train, or airplane accident

7. Have you ever had a life-threatering ever involved in a serious motor vehicle accident which required medical attention or that badly injured or killed someone?

3. Were you ever involved in any 'Other' accident where you or someone else was badly hurt? (e.g., plane crash,

3. A really bad accident at work or home drowning/nearly drowning, electrical or machinery accident, explosion, home fire, chemical leak, overexposure to radiation or toxic chemicals)

4. A hurricane, flood, earthquake, tornado, or fire 1. Have you ever experienced a natural disaster? (been caught in a flood, hurricane, earthquake).

12. While you were growing up, were you ever physically

5. Hit or kicked hard enough to injure - as a child punished in a way that resulted in bruises, burns, cuts, or broken bones?

9. Have you ever been hit/ beaten up and badly hurt by a stranger or by someone you didn't know well?

6. Hit or kicked hard enough to injure - as an adult

OR 14. Have you ever been slapped, punched, kicked, beaten up, or otherwise physically hurt by your spouse (or former spouse), a boyfriend/ girlfriend, or some other intimate partner?

15. Before your 13th birthday, did anyone who was at least 5 years older than you ever touch/ fondle your body in a sexual way, or make you touch/ fondle them in a sexual way?

OR 16. Before your 13th birthday, did anyone close to

7. Forced or made to have sexual contact - as a child your age ever touch you in a sexual way or make you touch them in a sexual way against your will or without your consent?

OR 17. After your 13th birthday and before your 18th birthday, did anyone touch the sexual parts of your body or make you touch the sexual parts of their body against your will or without your consent?

8. Forced or made to have sexual contact - as an adult 18. After your 18th birthday, did anyone touch the sexual parts of your body or make you touch the sexual parts of their body against your will or without your consent?

9. Attacked with a gun, knife, or weapon 11. Has anyone ever threatened to kill you or cause you serious physical harm?

4. Have you lived, worked, or had military service in a war

10. During military service - saw something horrible or was badly scared zone and been exposed to warfare or combat? (e.g., been in the vicinity of a rocket attack, people being fired upon, seeing someone get wounded or killed).

11. Sudden death of close family member or friend 5. Have you experienced the sudden, unexpected death of a close friend or loved one?

12. Seeing someone die suddenly or get badly hurt or killed

10. Have you ever seen someone else attacked and seriously injured or killed?

23. Have you experienced (or seen) any other events that were life threatening, caused serious injury, or were highly

15. Some other sudden event that made you feel very scared, helpless, or horrified disturbing or distressing? (e.g., lost in the wilderness, a serious animal bite, violent death of a pet, being kidnapped or held hostage, seeing a mutilated body or body parts). 
Table S2. Derivation of lifetime trauma history scores from items probing common trauma types across the Trauma History Screen (THS) and Traumatic Life Events Questionnaire (TLEQ) measures. For each of the 13 items (12 kinds of common trauma and one item probing experience other highly distressing events), participants scored 1 if they endorsed a particular event over their lifetime, and 0 otherwise (frequency-weighted response items from the TLEQ were collapsed to scores of 0 [never] or 1 [more than once]). Where multiple TLEQ items were deemed to be equivalent to a single THS item (THS items 6 and 7), endorsing any of the equivalent TLEQ items resulted in a score of 1 for that trauma type. Items from either scale that asked about events not probed in the other scale (e.g., sudden abandonment by partner or family from the THS, experience of miscarriage from the TLEQ) were not included - therefore this score may not represent complete trauma load for some participants. Across all participants, the mean score on this measure was 4.9 (SD 2.5, range 0-11, maximum possible score 13). 


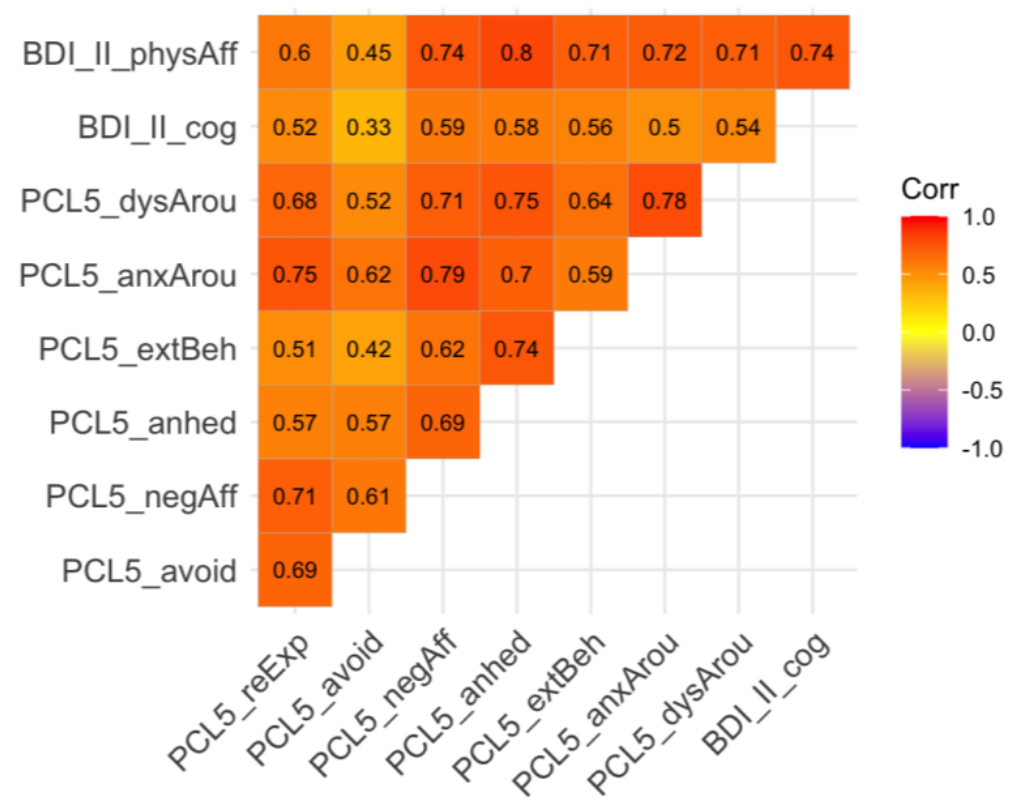

Figure S1. Correlation matrix for PTSD and depression symptom dimensions. Values represent magnitude of Pearson correlations between pairs of subscores, across all study participants. PCL5, PTSD Checklist for DSM-5; BDI_II, Beck Depression Inventory version II. PTSD symptom dimensions are defined as per Armour et al., 2015 and depression symptom dimensions are defined as per Bringmann et al., 2015. PCL-5_reExp, PCL-5 reexperiencing symptoms; PCL5_avoid, PCL-5 avoidance symptoms; PCL5_negAffect, PCL-5 negative affect; PCL5 anhed, PCL-5 anhedonia; PCL5_extBeh, PCL-5 externalizing behaviours; PCL5_anxArou, PCL-5 anxious arousal; PCL5_dysArou, PCL-5 dysphoric arousal; BDI_II_cog, BDI-II cognitive symptoms; BDI_II_physAff, BDI-II physical/affective symptoms. 
a

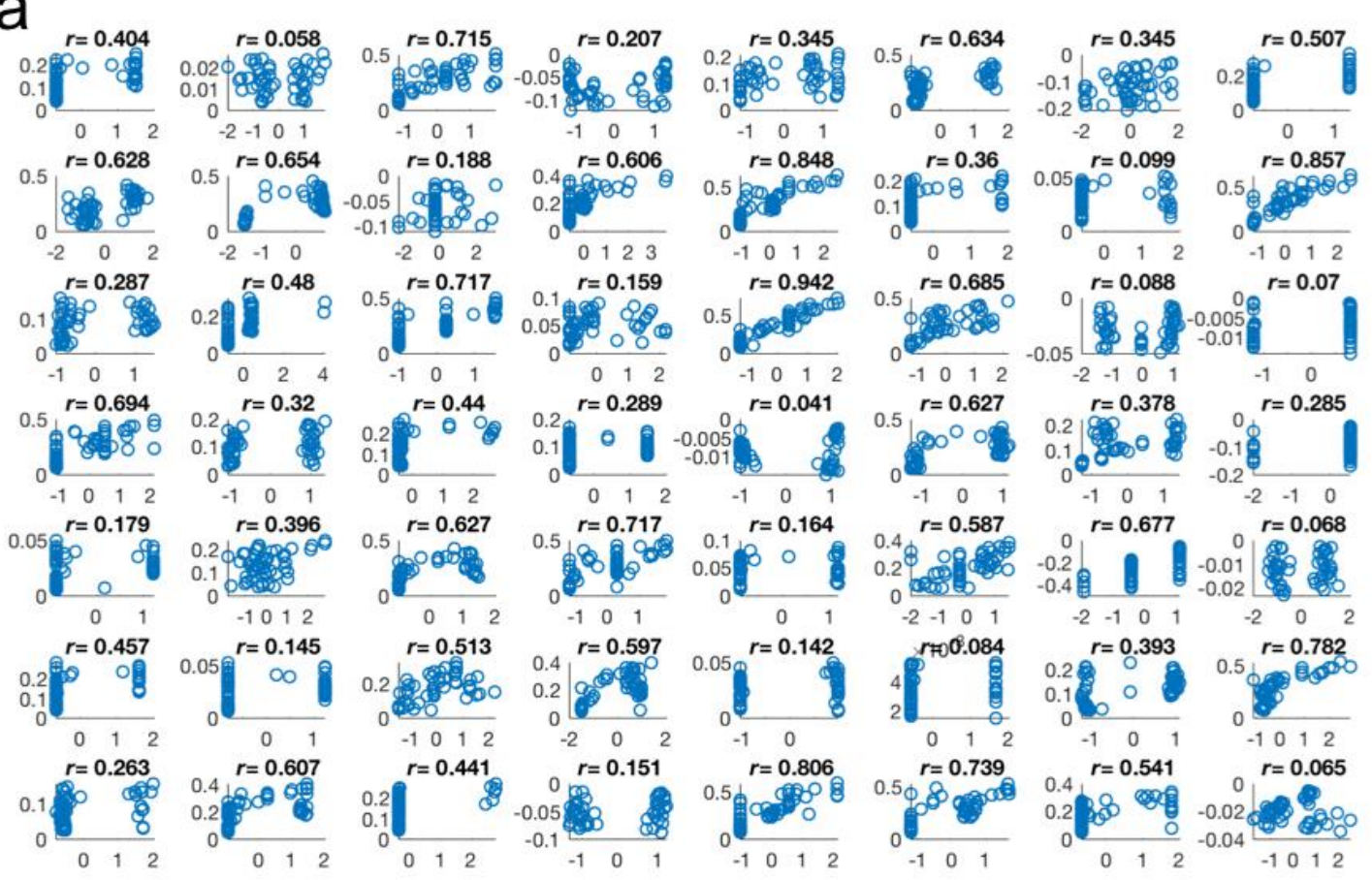

b

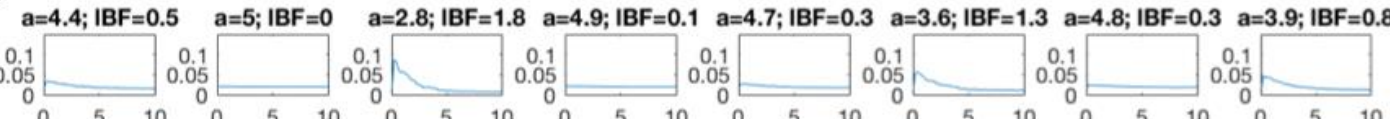
$a=3.7 ;$ IBF=1.1 $a=3.1 ;$ IBF=1.5 $\quad a=4.9 ; I B F=0 \quad a=3.7 ; I B F=0.8 \quad a=2.2 ; I B F=2.6 \quad a=4.6 ; I B F=0.3 \quad a=5 ; I B F=-0.1 \quad a=2.4 ; I B F=2.4$

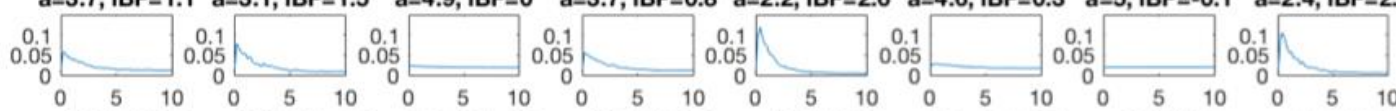
$a=4.5 ; I B F=0.2 \quad a=4.1 ; I B F=0.8 \quad a=2.9 ; I B F=1.8 \quad a=4.9 ; I B F=0.1 \quad a=1.9 ; I B F=3 \quad a=3.1 ; I B F=1.6 \quad a=5 ; I B F=0 \quad a=5 ; I B F=0$ 0.1
0.05 $a=2.8 ; I B F=1.7 \quad a=4.7 ; I B F=0.3 \quad a=4.5 ; I B F=0.6 \quad a=4.6 ; I B F=0.3 \quad a=5 ; I B F=0 \quad a=3.1 ; I B F=1.4 \quad a=4.6 ; I B F=0.5 \quad a=4.7 ; I B F=0.1$

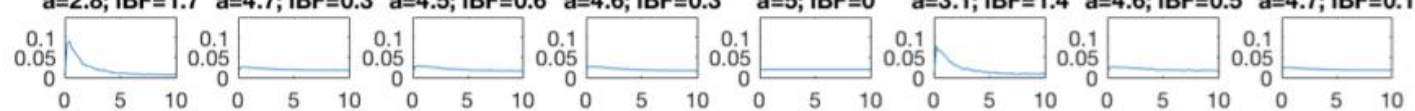

$a=4.8 ; I B F=-0.2 \quad a=4.4 ; I B F=0.4 \quad a=3.4 ; I B F=1.3 \quad a=2.8 ; I B F=1.8 \quad a=4.8 ; I B F=0.1 \quad a=3.7 ; I B F=1.2 \quad a=2.9 ; I B F=1.6 \quad a=5 ; I B F=0$

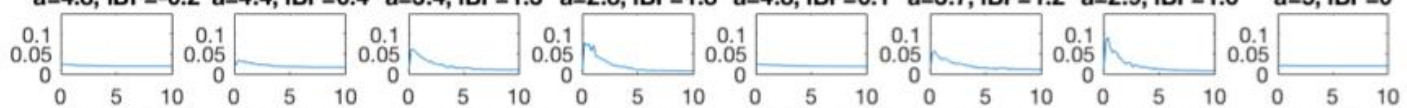

$a=4.2 ; I B F=0.7 \quad a=4.9 ; I B F=-0.1 \quad a=3.9 ; I B F=0.9 \quad a=3.1 ; I B F=1.2 \quad a=4.9 ; I B F=-0.1 \quad a=5 ; I B F=0 \quad a=4.1 ; I B F=0.4 \quad a=3.3 ; I B F=1.3$

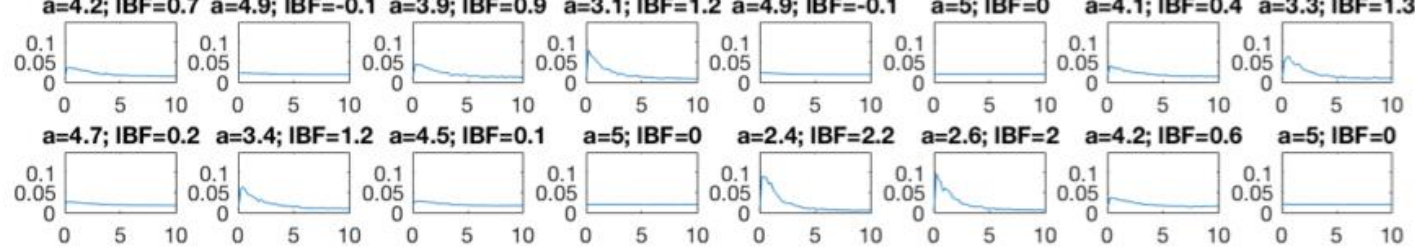

Figure S2. Latent cause model output. a Actual vs predicted loss expectancy ratings for each participant, as generated using the latent cause model of contextual extinction task data. $\mathrm{x}$ axis, actual ratings data (z-scored withinparticipants); y axis, predicted rating on each trial generated by the latent cause model. $r$ values represent Pearson correlation coefficients between actual and predicted values for each participant. b Posterior distribution over alpha values for each participant. $\mathrm{x}$ axis, alpha value, $\mathrm{y}$ axis, posterior probability density (the prior was a uniform probability distribution over the interval $[0,10])$. a, posterior alpha estimate; $1 \mathrm{BF}, \log \mathrm{BF}$, or likelihood of a model where alpha $>0$ compared one where alpha $=0$. 


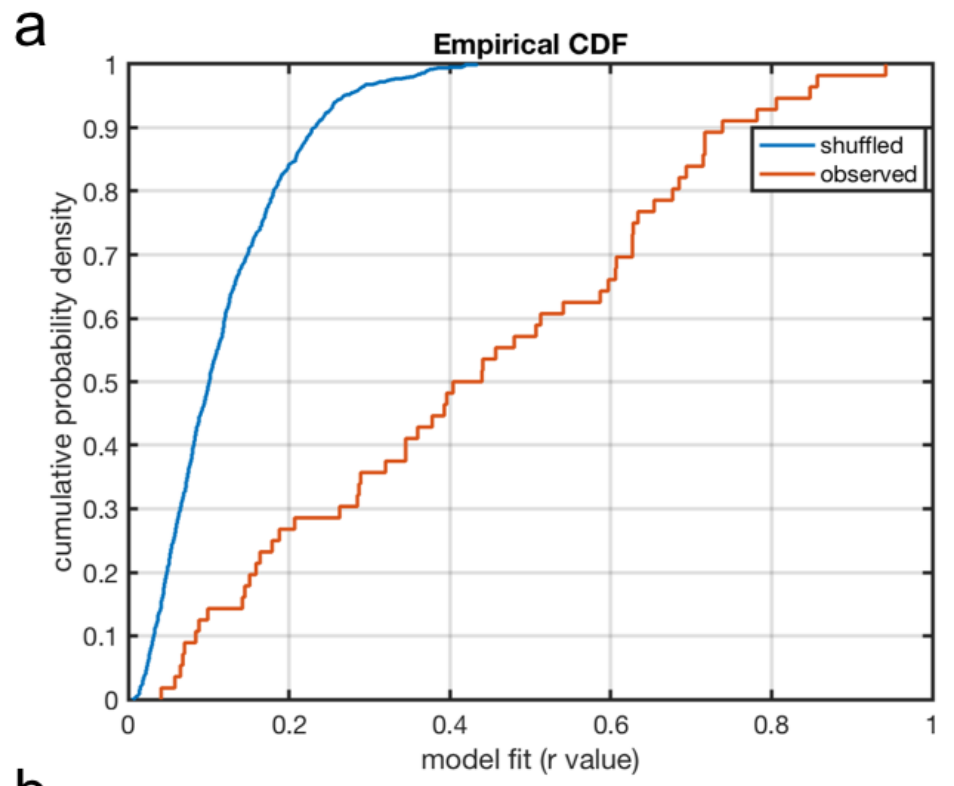

b

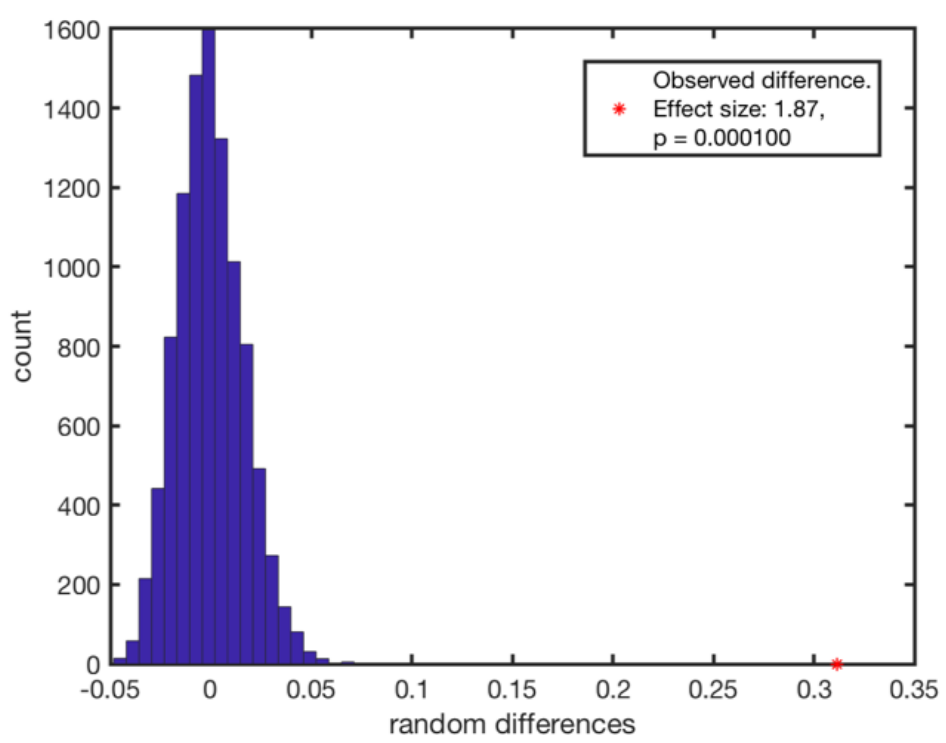

Figure S3. Comparison of observed model fit values to those generated from randomly shuffled ratings data. a In order to compare model fit $(r)$ values from observed data to randomly generated responses, $N=1000$ dummy datasets were generated by random permutation (shuffling) of observed ratings data. These ratings data were then submitted to latent cause modelling and the model output compared to actual values in the same way as for the real data. $\mathbf{b}$ A permutation difference test was then used to formally compare the means of two distributions. 
a

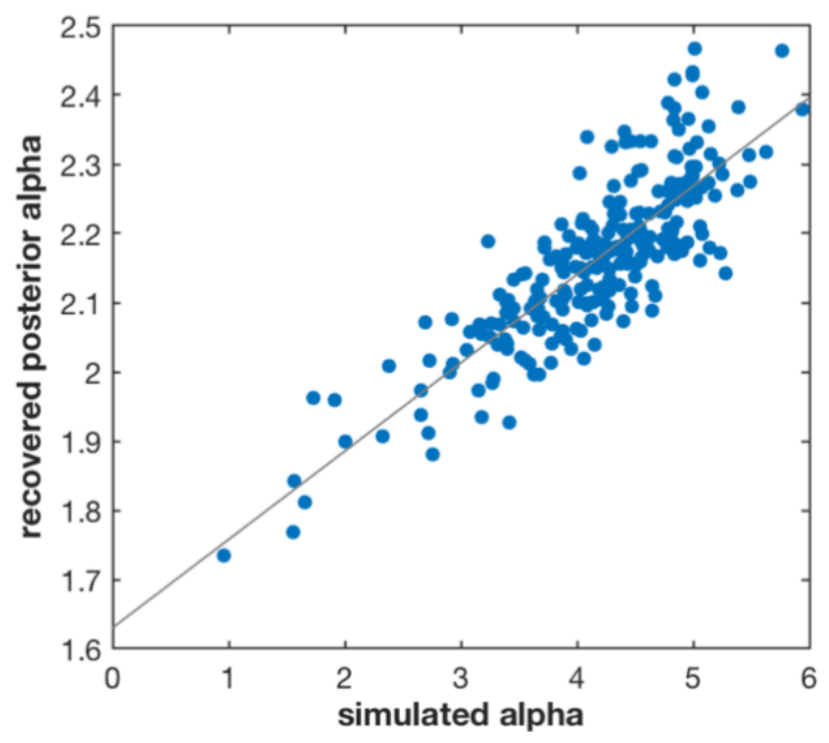

b

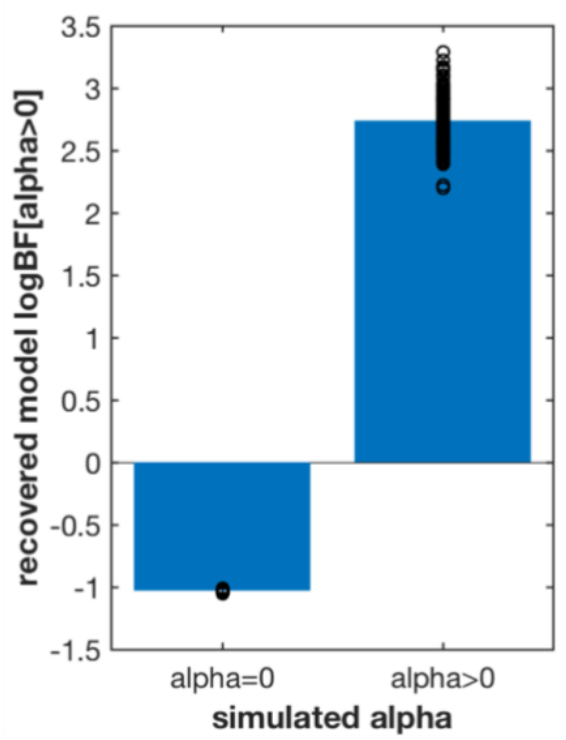

Figure S4. Simulation and recovery analysis. a Simulated $v$ s recovered posterior alpha values, for $N=250$ simulated task datasets, with alpha and beta values drawn randomly from the posterior distribution of these parameters across participants. For each simulation, alpha and beta point estimates were used to simulate responses to the same sequence of task stimuli and outcomes as that observed by study participants. The latent cause model was then fit to simulated data using the same procedure as in the main analysis: by fitting models with a range of alpha values (with uniform prior probability) to responses, then updating the posterior probability distribution for alpha according to the likelihood of each of these models (see Figure S2b). b Comparison of $\operatorname{logBF}[a l p h a>0$ ] scores generated for each simulated dataset, for datasets generated using alpha $=0$ (enriched with an additional $N=25$ simulations, to ensure adequate sampling), vs datasets where alpha was $>0$. Circles represent alpha and $\operatorname{logBF}$ values (respectively) recovered for individual simulated datasets. 


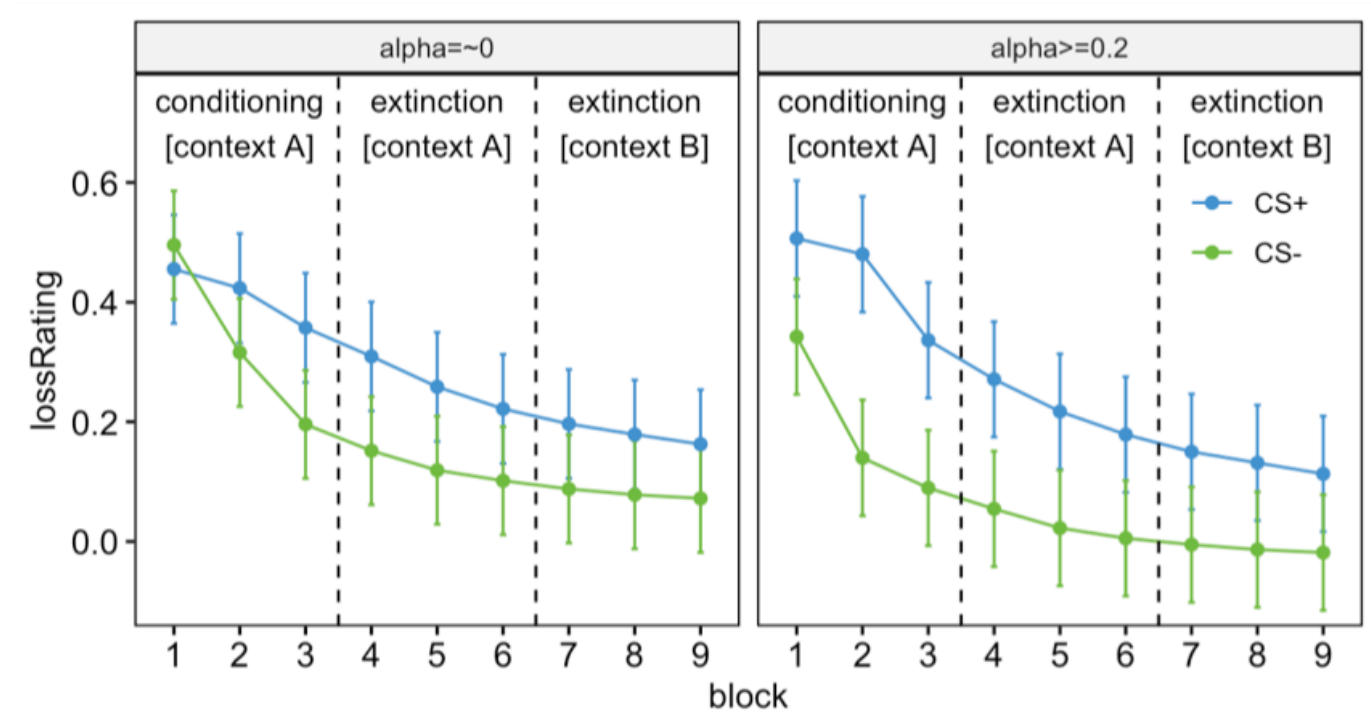

Figure S5. Loss expectancy ratings data from simulated datasets where alpha was constrained to be close to 0 vs simulated datasets where alpha was constrained to be $>\mathbf{0}$. Left panel, $N=100$ datasets, mean alpha $=0.0015 \pm 0.001$, mean recovered $\log B F$ over modelled period $=-0.39 \pm 0.62$; right panel, $N=100$ datasets, mean alpha $=1.13 \pm 1.1$, mean recovered $\log B F$ over modelled period $=0.42 \pm 0.05$. Since we observed relative invariance to the context change manipulation in our experimental data, for the purposes of these simulations, the change in context was not included in the model. Error bars represent standard error of the mean, and loss ratings are in arbitrary units (for all simulations depicted here, the scaling parameter beta was held constant at 1.0). 


\section{a}

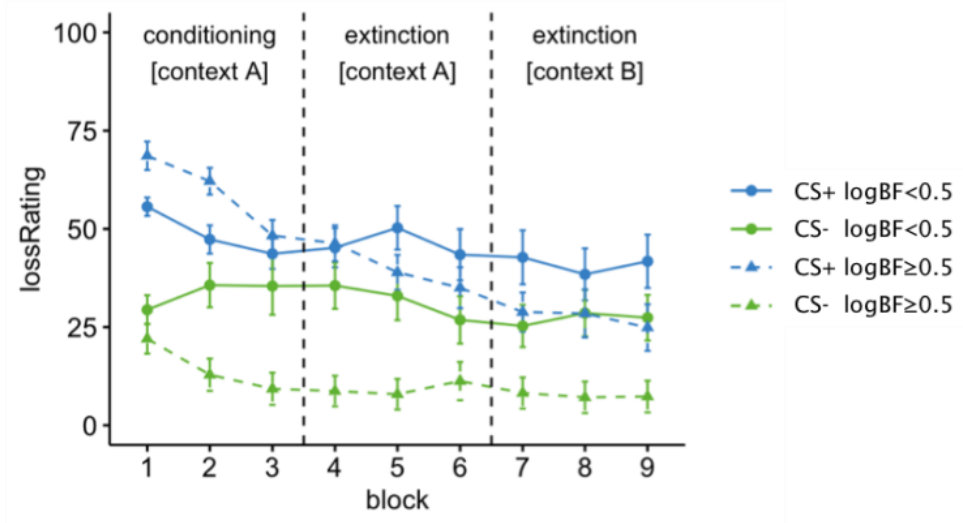

b

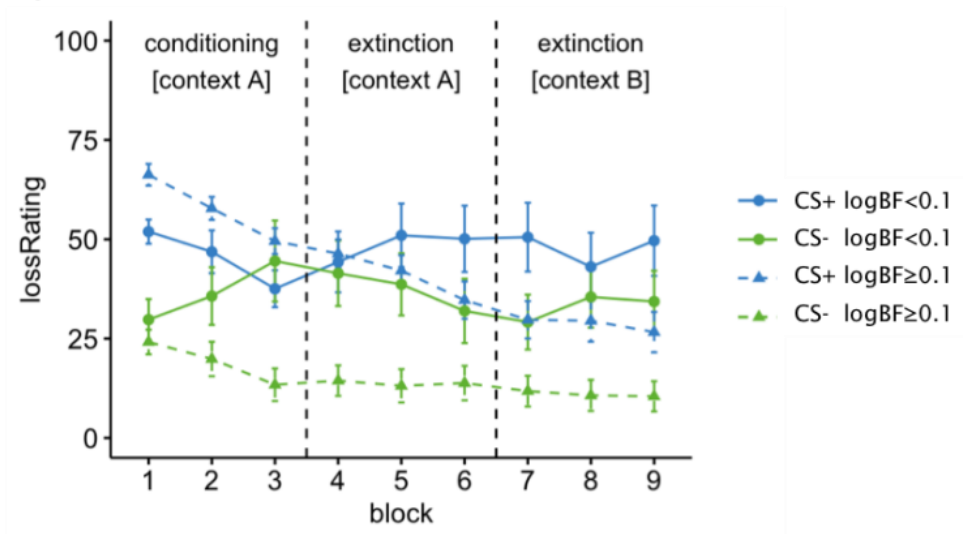

Figure S6. Differences in behaviour on the extinction learning task, according to posterior probability of a multi-cause model (logBF group) at two alternative thresholds for group definition. a $\operatorname{LogBF}[a l p h a>0] \geq 0.5$ ( $N=27$ vs $N=29$ participants). $\mathbf{b} \operatorname{LogBF}[a l p h a>0] \geq 0.1$ ( $N=39$ vs $N=17$ participants). In both cases, participants in the lower $\log \mathrm{BF}$ group exhibited slower extinction learning (flatter CS+ loss ratings traces) and less discrimination between aversively conditioned (CS+) and non aversively-conditioned (CS-) stimuli. Error bars represent standard error of the mean. 

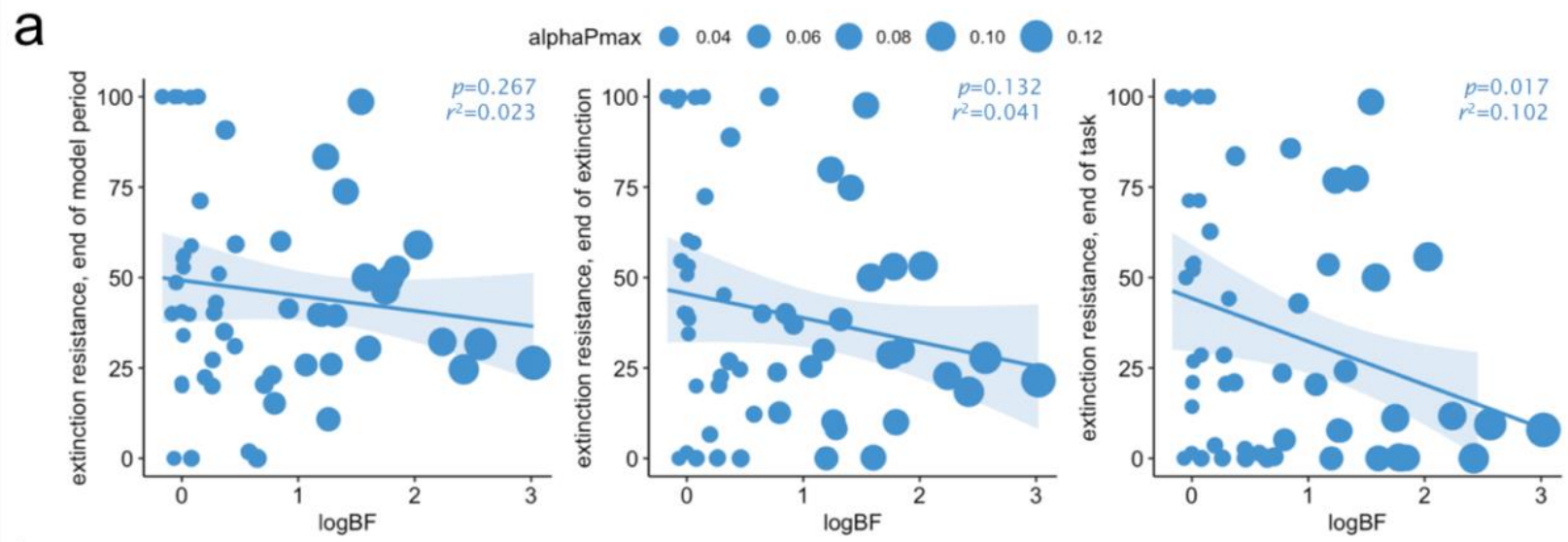

b
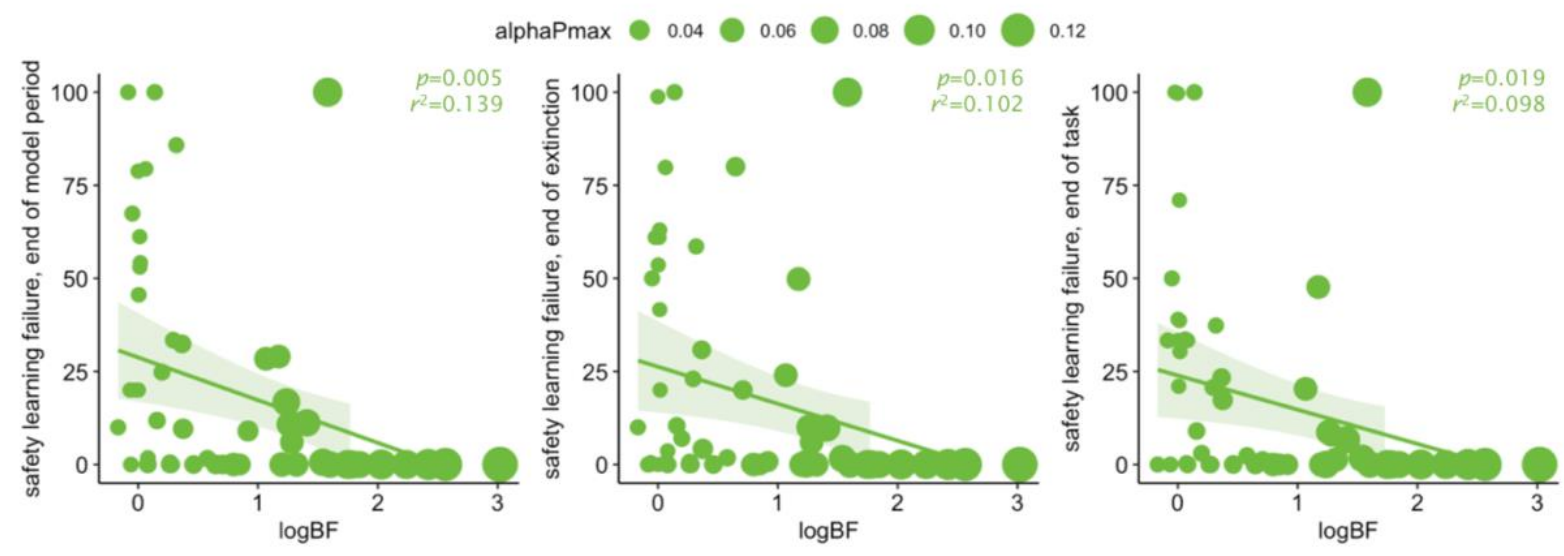

C
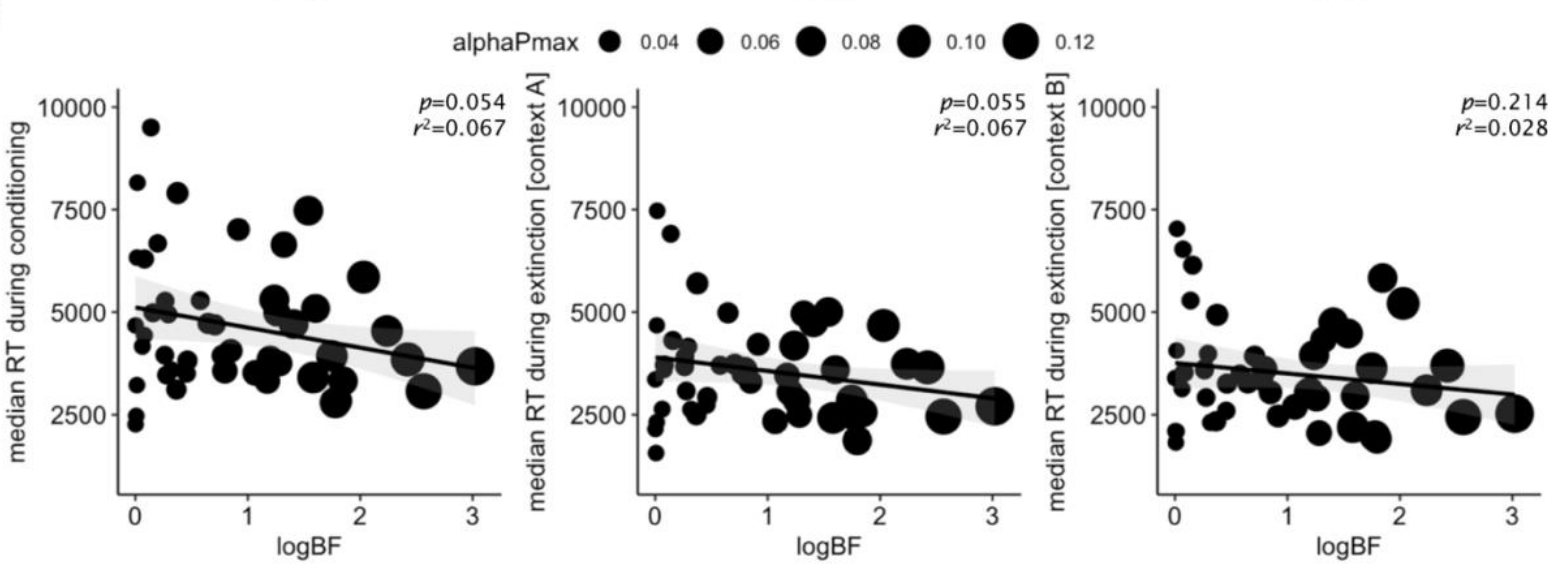

Figure S7. Relationships between individual differences in latent cause inference and extinction resistance, safety learning, and response times on the contextual extinction task. a Relationship between $\operatorname{logBF}$ values (probability of a model where alpha $>0$, compared to a single cause or alpha $=0$ model), estimated from initial conditioning and first two blocks of extinction, and resistance to extinction index (mean residual CS+ loss expectancy rating) at the end of the modelled period (second block of extinction, block 5), end of extinction (block 6), and end of the task (block 9). b Relationship between $\operatorname{logBF}$ values and over-generalization or safety learning failure (CS- loss expectancy ratings, in the absence of any association between the CS- and the loss outcome) at the end of the modelled period (second block of extinction, block 5), end of extinction (block 6), and end of the task (block 9). $\mathbf{c}$ Relationship between $\log \mathrm{BF}$ values and median response times (RTs) in ms to enter ratings during conditioning, initial extinction [in context $\mathrm{A}$ ], and further extinction [in novel context $\mathrm{B}$ ] stages. Regression lines represent linear model fit, weighted by posterior certainty in alpha parameter estimate (alphaPmax; higher certainty $=$ larger dot size). 

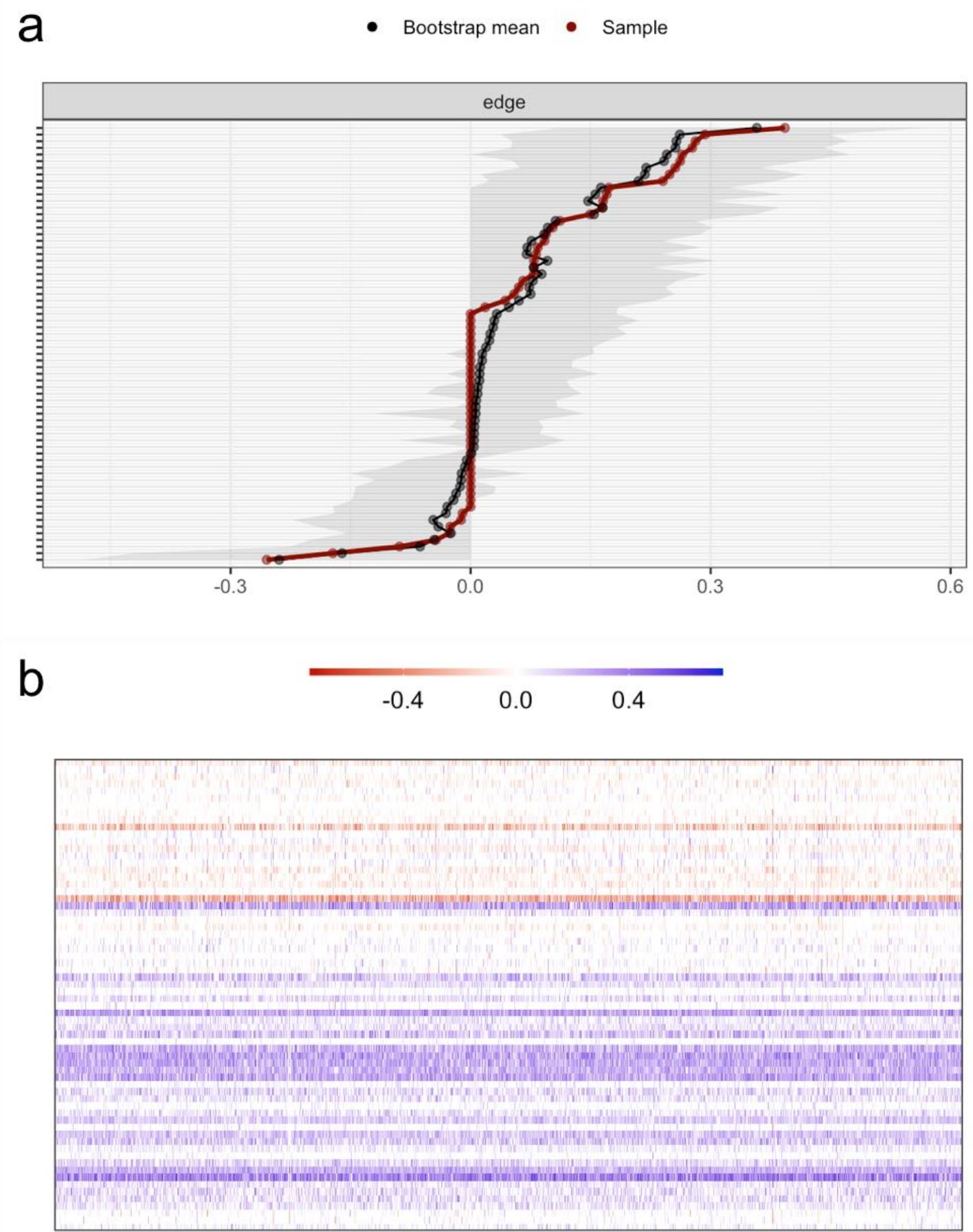

Figure S8. Results of bootstrap analysis of edge strength accuracy for the network incorporating extinction task metrics with PTSD and depression symptom dimensions. a Results of network stability analysis via nonparametric bootstrapping of edge weights ( $N=2000$ bootstraps). Each horizontal line represents one edge in the network, ordered from highest edge-weight to lowest edge-weight. The red line represents edge weight values in the same, the black line the mean value across bootstraps, and the gray area 95\% bootstrapped CIs for each edge. $\mathbf{b}$ Multiverse plot representation of the same data as in $a$. Every row indicates an edge, and every column represents a bootstrap, with colour and intensity proportional to edge strength. Figures generated using the R package bootnet (https://cran.r-project.org/package=bootnet). 
a

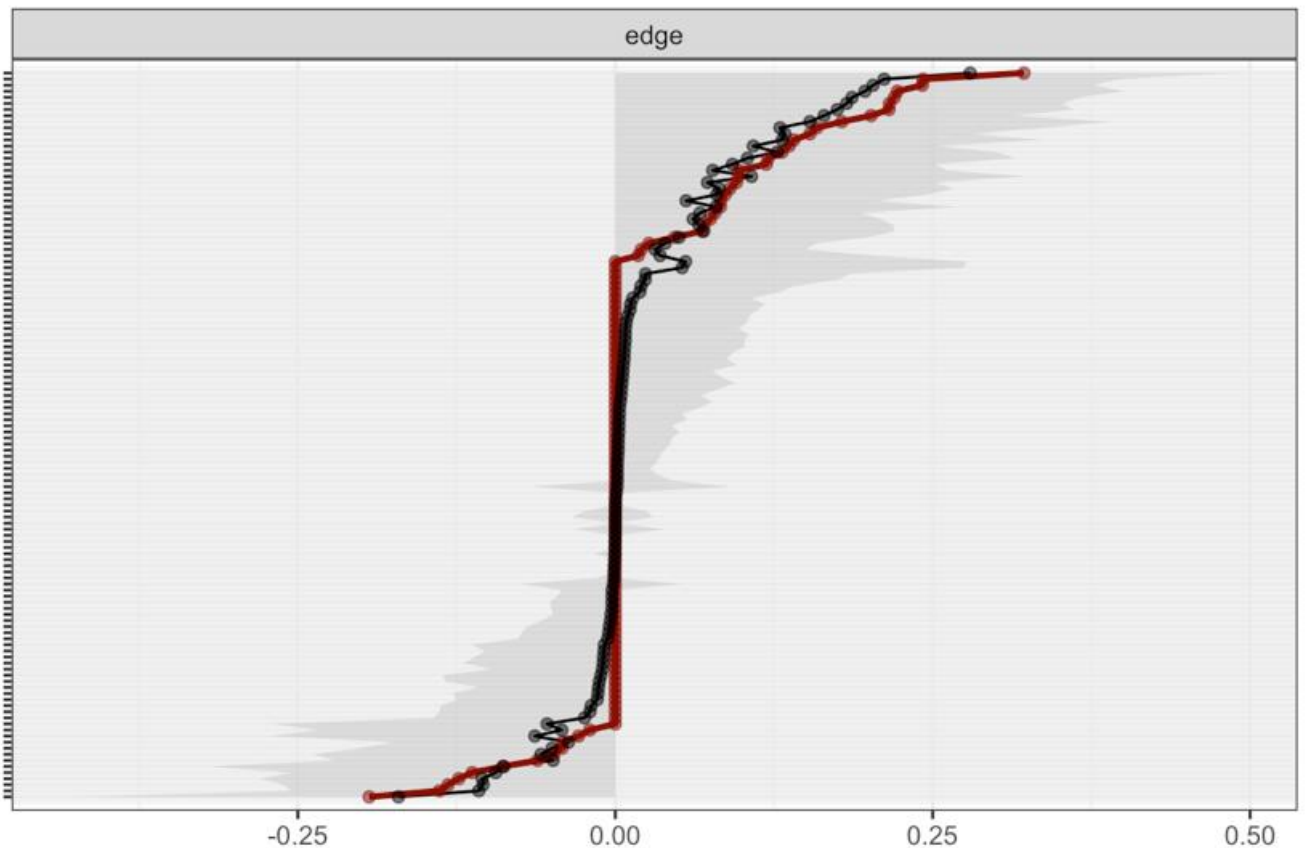

b
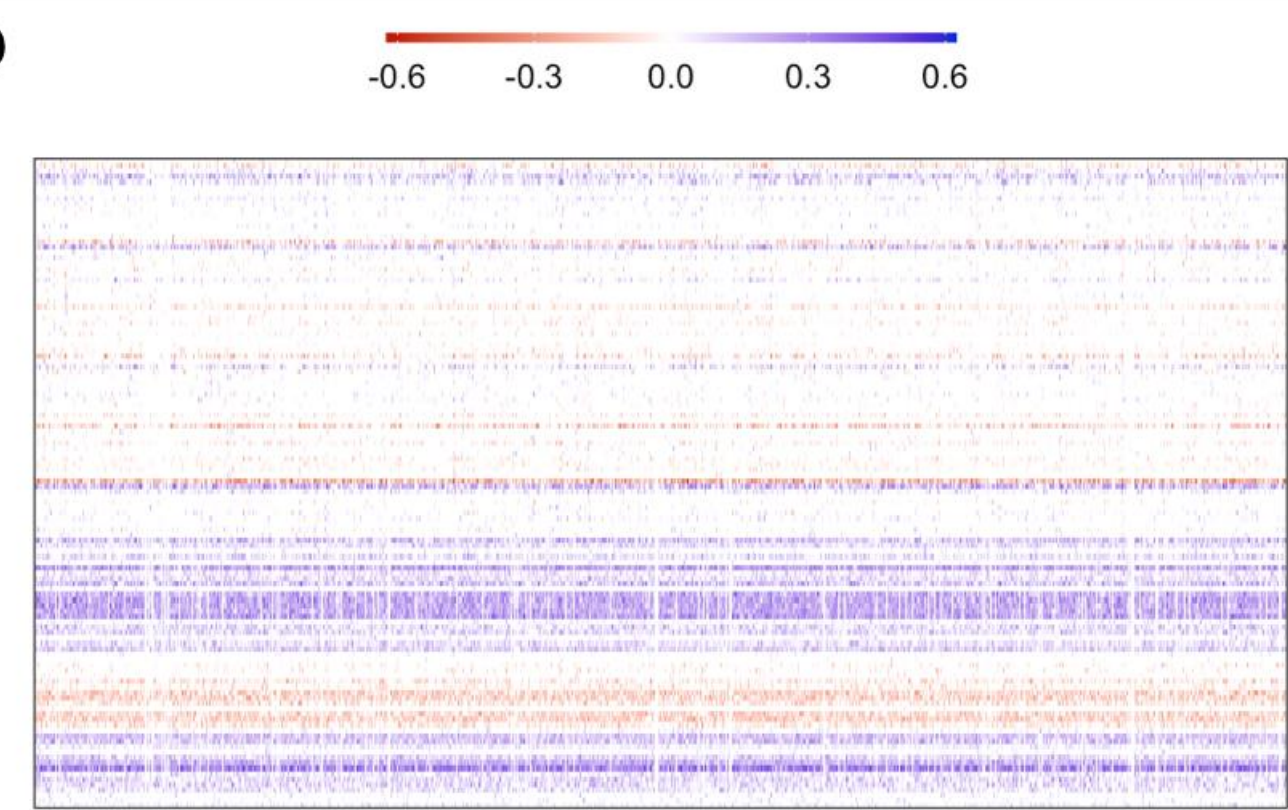

Figure S9. Results of bootstrap analysis of edge strength accuracy for the network incorporating extinction task metrics with PTSD and depression symptom dimensions, and other sociodemographic information. a Results of network stability analysis via nonparametric bootstrapping of edge weights ( $N=2000$ bootstraps). Each horizontal line represents one edge in the network, ordered from highest edge-weight to lowest edge-weight. The red line represents edge weight values in the same, the black line the mean value across bootstraps, and the gray area 95\% bootstrapped CIs for each edge. $\mathbf{b}$ Multiverse plot representation of the same data as in $a$. Every row indicates an edge, and every column represents a bootstrap, with colour and intensity proportional to edge strength. Figures generated using the R package bootnet (https://cran.r-project.org/package=bootnet). 


\section{Supplementary References}

Armour, C., Tsai, J., Durham, T. A., Charak, R., Biehn, T. L., Elhai, J. D., \& Pietrzak, R. H. (2015). Dimensional structure of DSM-5 posttraumatic stress symptoms: Support for a hybrid Anhedonia and Externalizing Behaviors model. Journal of Psychiatric Research, 61, 106-113. https://doi.org/10.1016/j.jpsychires.2014.10.012

Bringmann, L. F., Lemmens, L. H. J. M., Huibers, M. J. H., Borsboom, D., \& Tuerlinckx, F. (2015). Revealing the dynamic network structure of the Beck Depression Inventory-II. Psychological Medicine, 45(4), 747-757. https://doi.org/10.1017/S0033291714001809

Carlson, E. B., Smith, S. R., Palmieri, P. A., Dalenberg, C., Ruzek, J. I., Kimerling, R., Burling, T. A., \& Spain, D. A. (2011). Development and Validation of a Brief Self-Report Measure of Trauma Exposure: The Trauma History Screen. Psychological Assessment, 23(2), 463-477. https://doi.org/10.1037/a0022294

Chen, C., Salim, R., Rodriguez, J., Singh, R., Schechter, C., Dasaro, C. R., Todd, A. C., Crane, M., Moline, J. M., Udasin, I. G., Harrison, D. J., Luft, B. J., Southwick, S. M., Pietrzak, R. H., \& Feder, A. (2020). The Burden of Subthreshold Posttraumatic Stress Disorder in World Trade Center Responders in the Second Decade After 9/11. The Journal of Clinical Psychiatry, 81(1). https://doi.org/10.4088/JCP.19m12881

de Leeuw, J. R. (2015). jsPsych: A JavaScript library for creating behavioral experiments in a Web browser. Behavior Research Methods, 47(1), 1-12. https://doi.org/10.3758/s13428-014-0458-y

Epskamp, S., Borsboom, D., \& Fried, E. I. (2018). Estimating psychological networks and their accuracy: A tutorial paper. Behavior Research Methods, 50(1), 195-212. https://doi.org/10.3758/s13428-017-0862-1

Epskamp, S., Cramer, A. O. J., Waldorp, L. J., Schmittmann, V. D., \& Borsboom, D. (2012). qgraph: Network Visualizations of Relationships in Psychometric Data. Journal of Statistical Software, 048(i04). https://econpapers.repec.org/article/jssjstsof/v_3a048_3ai04.htm

Epskamp, S., \& Fried, E. I. (2018). A tutorial on regularized partial correlation networks. Psychological Methods, 23(4), 617-634. https://doi.org/10.1037/met0000167

Faelens, L., Hoorelbeke, K., Fried, E., De Raedt, R., \& Koster, E. H. W. (2019). Negative influences of Facebook use through the lens of network analysis. Computers in Human Behavior, 96, 1322. https://doi.org/10.1016/j.chb.2019.02.002

Feder, A., Mota, N., Salim, R., Rodriguez, J., Singh, R., Schaffer, J., Schechter, C. B., Cancelmo, L. M., Bromet, E. J., Katz, C. L., Reissman, D. B., Ozbay, F., Kotov, R., Crane, M., Harrison, D. J., Herbert, R., Levin, S. M., Luft, B. J., Moline, J. M., .. Pietrzak, R. H. (2016). Risk, coping and PTSD symptom trajectories in World Trade Center responders. Journal of Psychiatric Research, 82, 68-79. https://doi.org/10.1016/j.jpsychires.2016.07.003

Foygel, R., \& Drton, M. (2010). Extended Bayesian Information Criteria for Gaussian Graphical Models. In J. D. Lafferty, C. K. I. Williams, J. Shawe-Taylor, R. S. Zemel, \& A. Culotta (Eds.), Advances in Neural Information Processing Systems 23 (pp. 604-612). Curran Associates, Inc. http://papers.nips.cc/paper/4087-extended-bayesian-information-criteria-for-gaussiangraphical-models.pdf

Fried, E. I., \& Burger, J. (2019). 2019-09 Workshop-Network analysis workshop (FLAMES, Ghent). https://doi.org/10.17605/OSF.IO/P527F 
Friedman, J., Hastie, T., \& Tibshirani, R. (2008). Sparse inverse covariance estimation with the graphical lasso. Biostatistics, 9(3), 432-441. https://doi.org/10.1093/biostatistics/kxm045

Fritz, J., Fried, E. I., Goodyer, I. M., Wilkinson, P. O., \& van Harmelen, A.-L. (2018). A Network Model of Resilience Factors for Adolescents with and without Exposure to Childhood Adversity. Scientific Reports, 8(1), 15774. https://doi.org/10.1038/s41598-018-34130-2

Fruchterman, T. M. J., \& Reingold, E. M. (1991). Graph drawing by force-directed placement. Software: Practice and Experience, 21(11), 1129-1164. https://doi.org/10.1002/spe.4380211102

Gershman, S. J., \& Hartley, C. A. (2015). Individual differences in learning predict the return of fear. Learning \& Behavior, 43(3), 243-250. https://doi.org/10.3758/s13420-015-0176-z

Gershman, S. J., \& Niv, Y. (2012). Exploring a latent cause theory of classical conditioning. Learning \& Behavior, 40(3), 255-268. https://doi.org/10.3758/s13420-012-0080-8

Jones, P. J., Mair, P., \& McNally, R. J. (2018). Visualizing Psychological Networks: A Tutorial in R. Frontiers in Psychology, 9. https://doi.org/10.3389/fpsyg.2018.01742

Karam, E. G., Friedman, M. J., Hill, E. D., Kessler, R. C., McLaughlin, K. A., Petukhova, M., Sampson, L., Shahly, V., Angermeyer, M. C., Bromet, E. J., Girolamo, G. de, Graaf, R. de, Demyttenaere, K., Ferry, F., Florescu, S. E., Haro, J. M., He, Y., Karam, A. N., Kawakami, N., ... Koenen, K. C. (2014). Cumulative Traumas and Risk Thresholds: 12-Month PTSD in the World Mental Health (WMH) Surveys. Depression and Anxiety, 31(2), 130-142. https://doi.org/10.1002/da.22169

Kass, R. E., \& Raftery, A. E. (1995). Bayes Factors. Journal of the American Statistical Association, 90(430), 773-795. https://doi.org/10.1080/01621459.1995.10476572

Kubany, E. S., Haynes, S. N., Leisen, M. B., Owens, J. A., Kaplan, A. S., Watson, S. B., \& Burns, K. (2000). Development and preliminary validation of a brief broad-spectrum measure of trauma exposure: The Traumatic Life Events Questionnaire. Psychological Assessment, 12(2), 210-224. https://doi.org/10.1037//1040-3590.12.2.210

Maruff, P., Thomas, E., Cysique, L., Brew, B., Collie, A., Snyder, P., \& Pietrzak, R. H. (2009). Validity of the CogState Brief Battery: Relationship to Standardized Tests and Sensitivity to Cognitive Impairment in Mild Traumatic Brain Injury, Schizophrenia, and AIDS Dementia Complex. Archives of Clinical Neuropsychology, 24(2), 165-178. https://doi.org/10.1093/arclin/acp010

McLaughlin, K. A., Koenen, K. C., Friedman, M. J., Ruscio, A. M., Karam, E. G., Shahly, V., Stein, D. J., Hill, E. D., Petukhova, M., Alonso, J., Andrade, L. H., Angermeyer, M. C., Borges, G., de Girolamo, G., de Graaf, R., Demyttenaere, K., Florescu, S. E., Mladenova, M., PosadaVilla, J., ... Kessler, R. C. (2015). Subthreshold Posttraumatic Stress Disorder in the World Health Organization World Mental Health Surveys. Biological Psychiatry, 77(4), 375-384. https://doi.org/10.1016/j.biopsych.2014.03.028

Orederu, T., \& Schiller, D. (2018). Fast and slow extinction pathways in defensive survival circuits. Current Opinion in Behavioral Sciences, 24, 96-103. https://doi.org/10.1016/j.cobeha.2018.06.004

Sherbourne, C. D., \& Stewart, A. L. (1991). The MOS social support survey. Social Science \& Medicine, 32(6), 705-714. https://doi.org/10.1016/0277-9536(91)90150-B 
Stevens, J. S., \& Jovanovic, T. (2019). Role of social cognition in post-traumatic stress disorder: A review and meta-analysis. Genes, Brain and Behavior, 18(1), e12518. https://doi.org/10.1111/gbb.12518

Tibshirani, R. (1996). Regression Shrinkage and Selection via the Lasso. Journal of the Royal Statistical Society. Series B (Methodological), 58(1), 267-288. https://doi.org/10.2307/2346178

Zhao, T., Liu, H., Roeder, K., Lafferty, J., \& Wasserman, L. (2012). The huge Package for Highdimensional Undirected Graph Estimation in R. Journal of Machine Learning Research, 13(Apr), 1059-1062. 